\title{
A SHORT SURVEY ON THE INTEGRAL IDENTITY CONJECTURE AND THEORIES OF MOTIVIC INTEGRATION
}

\author{
LÊ QUY THUONG
}

\begin{abstract}
In Kontsevich-Soibelman's theory of motivic Donaldson-Thomas invariants for three-dimensional noncommutative Calabi-Yau varieties, the integral identity conjecture plays a crucial role as it involves the existence of these invariants. A purpose of this note is to show how the conjecture arises. Because of the integral identity's nature we shall give a quick tour on theories of motivic integration, from which lead to a proof of the conjecture for ground fields algebraically closed of characteristic zero.
\end{abstract}

\section{INTRODUCTION}

Historically, Thomas in his thesis and his paper [31] introduced an invariant for a 3dimensional Calabi-Yau manifold $M$ as a counting invariant of coherent sheaves on $M$, which analogizes the Casson invariant on a real 3-dimensional manifold. This kind of invariant was then named after him and his advisor Donaldson. According to [16], the moduli space $\mathcal{M}$ of coherent sheaves on $M$ can be locally presented as the critical locus of a holomorphic Chern-Simons functional $f$. By this, a Donaldson-Thomas invariant for $M$ can be described as an integral of the Behrend function over the moduli space $\mathcal{M}$. In view of [1], the value of the Behrend function is defined in terms of the Euler characteristic of the Milnor fiber $F_{f}$ of the Chern-Simons functional $f$, from which the Donaldson-Thomas invariant arises. In [17], replacing the Milnor fiber by a so-called motivic Milnor fiber (defined by Denef-Loeser [8] using motivic integration) Kontsevich and Soibelman study motivic Donaldson-Thomas invariants for 3-dimensional Calabi-Yau manifolds. The theory of Kontsevich and Soibelman allows, under appropriate realizations (e.g., a cohomology functor), to obtain refinements of (classical) Donaldson-Thomas invariants. The motivic Donaldson-Thomas invariants are also realized physically as "BPS invariants". Among 3-dimensional Calabi-Yau categories, the derived category of coherent sheaves on a compact (or local) 3-dimensional Calabi-Yau manifolds is a central object.

Let us now give a brief review due to [17] and [18] on the direct elements in KontsevichSoibelman's theory concerning the integral identity conjecture. Let $\mathcal{C}$ be an ind-constructible triangulated $A_{\infty}$-category over a field $k$. For any strict sector $V$ in $\mathbb{R}^{2}$, we consider a collection of full subcategories $\mathcal{C}_{V}$ of $\mathcal{C}$. Then one can construct the motivic Hall algebra $H(\mathcal{C})$ as a graded associative algebra admitting for any strict sector $V$ an element $A_{V}^{\text {Hall }}$ invertible in a completion $\widehat{H}\left(\mathcal{C}_{V}\right)$ of $H\left(\mathcal{C}_{V}\right)$. More precisely, in terms of a countable decomposition $\operatorname{Ob}(\mathcal{C})=\sqcup_{i \in I} Y_{i}$ into

2010 Mathematics Subject Classification. Primary 03C60, 14B20, 14E18, 14G22, 32S45, 11S80.

Key words and phrases. motivic integration, formal schemes, rigid varieties, volume Poincaré series, resolution of singularity, integral identity conjecture, definable sets.

This research is funded by the Vietnam National University, Hanoi (VNU) under project number QG.16.06. This research is also supported by ERCEA Consolidator Grant 615655 - NMST and by the Basque Government through the BERC 2014-2017 program and by Spanish Ministry of Economy and Competitiveness MINECO: BCAM Severo Ochoa excellence accreditation SEV-2013-0323. 
constructible subsets such that $\mathrm{GL}\left(N_{i}\right)$ act on $Y_{i}$, respectively, we have

$$
A_{V}^{\mathrm{Hall}}=\sum_{i \in I} \mathbf{1}_{\left(\mathrm{Ob}\left(\mathcal{C}_{V}\right) \cap Y_{i}, \mathrm{GL}\left(N_{i}\right)\right)},
$$

where $\mathbf{1}_{S}$ is the identity function interpreted as a counting measure (see [17, Section 6.1]). One requires that $A_{V}^{\text {Hall }}$ must satisfy the factorization property that $A_{V}^{\text {Hall }}=A_{V_{1}}^{\text {Hall }} \cdot A_{V_{2}}^{\text {Hall }}$, where $V=V_{1} \sqcup V_{2}$ and the decomposition is taken clockwisely. These constructions also depend on a constructible stability condition on $\mathcal{C}$. By definition, a stability datum on the algebra $H(\mathcal{C})=\bigoplus_{\gamma \in \Gamma} H(\mathcal{C})_{\gamma}$ consists of a morphism of abelian groups $Z: \Gamma \rightarrow \mathbb{C}$, with $\Gamma$ a free abelian group endowed with an integer-valued bilinear form $\langle\bullet, \bullet\rangle$, and a collection $a=(a(\gamma))_{\gamma \in \Gamma \backslash\{0\}}$ with property that there exists a positive real number $c>0$ such that $\|\gamma\| \leq c|Z(\gamma)|$ for any $\gamma \in \Gamma$ with $a(\gamma) \neq 0$. In other words, a constructible stability condition on $\mathcal{C}$ is what guarantees that the set of stability data on $H(\mathcal{C})$ is the same as that of group elements in $H(\mathcal{C})$ having the factorization property.

Assume that the field $k$ has characteristic zero. It is obvious that the group schemes $\mu_{n}=\operatorname{Spec}\left(k[t] /\left(t^{n}-1\right)\right)$ and the maps $\xi \in \mu_{n p} \mapsto \xi^{p} \in \mu_{n}$ form a projective system, whose limit will be denoted by $\hat{\mu}$. Let $\operatorname{Var}_{k, \hat{\mu}}$ be the category of algebraic $k$-varieties $X$ endowed with a $\hat{\mu}$-action $\sigma$. The Grothendieck group $K_{0}\left(\operatorname{Var}_{k, \hat{\mu}}\right)$ is an abelian group generated by symbols $[X]=[X, \sigma]$ for $(X, \sigma)$ in $\operatorname{Var}_{k, \hat{\mu}}$ such that $[X]=[Y]$ whenever $X$ is $\hat{\mu}$-equivariantly isomorphic to $Y,[X]=[Y]+[X \backslash Y]$ for $Y$ Zariski closed in $X$ with the $\hat{\mu}$-action induced from $X$, and $[X \times V]=\left[X \times \mathbb{A}_{k}^{e}\right]$ if $V$ is an $e$-dimensional affine $k$-space with arbitrary linear $\hat{\mu}$-action and the action on $\mathbb{A}_{k}^{e}$ is trivial. Furthermore, $K_{0}\left(\operatorname{Var}_{k, \hat{\mu}}\right)$ has a ring structure with unit induced by the cartesian product. Denote by $\mathcal{M}_{k}^{\hat{\mu}}$ the localization $K_{0}\left(\operatorname{Var}_{k, \hat{\mu}}\right)\left[\mathbb{L}^{-1}\right]$, with $\mathbb{L}:=\left[\mathbb{A}_{k}^{1}\right]$. Let $C_{0}$ be a commutative ring with unit containing an invertible symbol $\mathbb{L}^{\frac{1}{2}}$, which is a square root of $\mathbb{L}$. We consider a $C_{0}$-linear associative algebra $\mathcal{R}_{\Gamma, C_{0}}$ generated by symbols $\widehat{e}_{\gamma}$, for $\gamma \in \Gamma$, modulo the usual conditions $\widehat{e}_{0}=1$ and $\widehat{e}_{\gamma_{1}} \widehat{e}_{\gamma_{2}}=\mathbb{L}^{\frac{1}{2}\left\langle\gamma_{1}, \gamma_{2}\right\rangle} \widehat{e}_{\gamma_{1}+\gamma_{2}}$. If we choose $C_{0}$ to be the ring $\mathcal{M}_{k, \text { loc }}^{\hat{\mu}}:=\mathcal{M}_{k}^{\hat{\mu}}\left[\left\{\left(1-\mathbb{L}^{i}\right)^{-1}\right\}_{i \in \mathbb{N}}\right]$, then $\mathcal{R}_{\Gamma}:=\mathcal{R}_{\Gamma, C_{0}}$ is the motivic quantum torus associated with $\Gamma$ (cf. [17, Section 6.2]).

According to [17], in the theory of motivic Donaldson-Thomas invariants for 3-dimensional Calabi-Yau categories, the map $\mathcal{D}: H(\mathcal{C}) \rightarrow \mathcal{R}_{\Gamma}$ defined by $\mathcal{D}(\nu)=(\nu, w) \widehat{e}_{\gamma}$, for $\nu \in H(\mathcal{C})_{\gamma}$, plays a central role (cf. [17, Theorem 8]). Here $w$ is the motivic weight and $(\bullet, \bullet)$ is the pairing between motivic measures and motivic functions in the sense of [17]. The expected event that the map $\mathcal{D}$ is a homomorphism of $\Gamma$-graded $\mathbb{Q}$-algebras in fact depends absolutely on the positiveness of the integral identity conjecture and the orientation data. Indeed, let us consider the elements $\nu_{E_{1}}$ and $\nu_{E_{2}}$ of $H(\mathcal{C})$ given by the classes of the morphisms pt $\mapsto E_{1} \in \mathrm{Ob}(\mathcal{C})$ and pt $\mapsto E_{2} \in \mathrm{Ob}(\mathcal{C})$. By choosing orientation data defined in [17, Section 5] and using the Calabi-Yau property and the motivic Thom-Sebastiani theorem (cf. [9], [23] and [20]), Kontsevich-Soibelman's computation of the images $\mathcal{D}\left(\nu_{E_{1}}\right), \mathcal{D}\left(\nu_{E_{2}}\right)$ and $\mathcal{D}\left(\nu_{E_{1}} \cdot \nu_{E_{2}}\right)$ in the motivic quantum torus ring $\mathcal{R}_{\Gamma}$ shows that $\mathcal{D}\left(\nu_{E_{1}}\right) \mathcal{D}\left(\nu_{E_{2}}\right)=\mathcal{D}\left(\nu_{E_{1}} \cdot \nu_{E_{2}}\right)$ if the following identity holds in the ring $\mathcal{M}_{k}^{\hat{\mu}}$ :

$$
\begin{aligned}
\mathbb{L}^{\operatorname{dim} \operatorname{Ext}^{1}\left(E_{2}, E_{1}\right)}( & \left.1-\mathcal{S}_{W_{E_{1}}^{\min } \oplus W_{E_{2}}^{\min }, 0}\right) \bar{I}\left(h\left(E_{1}\right)\right) \bar{I}\left(h\left(E_{2}\right)\right) \\
& =\int_{\alpha \in \operatorname{Ext}^{1}\left(E_{2}, E_{1}\right)} \mathbb{L}^{\frac{1}{2}\left(\left(E_{\alpha}, E_{\alpha}\right)_{1}-\left(E_{1} \oplus E_{2}, E_{1} \oplus E_{2}\right)_{1}\right)}\left(1-\mathcal{S}_{W_{E_{\alpha}}^{\min }, 0}\right) \bar{I}\left(h\left(E_{\alpha}\right)\right) .
\end{aligned}
$$

Here, for any $E \in \operatorname{Ob}(\mathcal{C}), W_{E}^{\text {min }}$ is the potential of a minimal model $\mathcal{C}^{\text {min }}$ of $\mathcal{C}$, which is a formal series in $\alpha \in \operatorname{Ext}^{1}(E, E)$, with $\operatorname{Ext}^{1}(E, E)$ possibly viewed as an algebraic $k$-variety 
(see [17, Section 3.3]). We denote by $\mathcal{S}_{\mathfrak{f}, x}$ the motivic Milnor fiber of a formal function $\mathfrak{f}$ at a closed point $x$ in its special fiber, this motivic object lives in $\mathcal{M}_{k}^{\hat{\mu}}$ and will be studied in most part of the note. Also, for $N \in \mathbb{Z}$, the truncated Euler characteristic $(E, F)_{\leq N}$ is defined to be $\sum_{i<N}(-1)^{i} \operatorname{dim} \operatorname{Ext}^{i}(E, F)$. Finally, the elements $\bar{I}\left(h\left(E_{i}\right)\right)$ and $\bar{I}\left(h\left(E_{\alpha}\right)\right)$ appear in the orientation data, which satisfy the main property of the orientation data on exact triangles. Processing all the information concerning the orientation data, it remains the following identity

$$
\int_{\alpha \in \operatorname{Ext}^{1}\left(E_{2}, E_{1}\right)} \mathcal{S}_{W_{E_{1}, E_{2}},(0, \alpha, 0,0)}=\mathbb{L}^{\operatorname{dim} \operatorname{Ext}^{1}\left(E_{2}, E_{1}\right)} \mathcal{S}_{\left.W_{E_{1}, E_{2}}\right|_{\operatorname{Ext}^{1}\left(E_{1}, E_{1}\right) \oplus \operatorname{Ext}^{1}\left(E_{2}, E_{2}\right)},(0,0)} .
$$

As described in Step 3 of the proof of [17, Theorem 8], the potential $W_{E_{1}, E_{2}}$ is a formal series $\mathfrak{f}(x, \alpha, \beta, y)$ in the graded vector space $\mathcal{M}_{E_{1}, E_{2}}$ defined as the abelianization of a series $\sum_{n \geq 3} W_{n} / n$ in cyclic paths in the quivers $Q_{E_{1}, E_{2}}$ with the vertices $E_{1}$ and $E_{2}$, and with $\operatorname{dim} \operatorname{Ext}^{1}\left(E_{i}, E_{j}\right)$ edges between $E_{i}$ and $E_{j}$, for $i, j \in\{1,2\}$. In such a cyclic path, both directions $E_{1} \rightarrow E_{2}$ and $E_{2} \rightarrow E_{1}$ have the same number of edges, thus the formal series $W_{E_{1}, E_{2}}$ is $\mathbb{G}_{m, k}$-invariant with respect to the $\mathbb{G}_{m, k}$-action on $\mathcal{M}_{E_{1}, E_{2}}$ with the weights wt $(x)=$ $\operatorname{wt}(y)=0, \operatorname{wt}(\alpha)=-\operatorname{wt}(\beta)=1$. Identifying $\operatorname{Ext}^{1}\left(E_{2}, E_{1}\right)=\mathbb{A}_{k}^{d_{1}}, \operatorname{Ext}^{1}\left(E_{1}, E_{2}\right)=\mathbb{A}_{k}^{d_{2}}$ and $\operatorname{Ext}^{1}\left(E_{1}, E_{1}\right) \oplus \operatorname{Ext}^{1}\left(E_{2}, E_{2}\right)=\mathbb{A}_{k}^{d_{3}}$, for some positive integers $d_{1}, d_{2}$ and $d_{3}$, the previous formula is rewritten as follows.

Conjecture (Kontsevich-Soibelman). With the previous notation and hypotheses, the identity

$$
\int_{\alpha \in \mathbb{A}_{k}^{d_{1}}} \mathcal{S}_{\mathfrak{f}, \alpha}=\mathbb{L}^{d_{1}} \mathcal{S}_{\mathfrak{f}_{\mathbb{A}_{k}}^{d_{3}}, 0}
$$

holds in $\mathcal{M}_{k}^{\hat{\mu}}$.

The identity in this conjecture is known as the integral identity (cf. [17, Conjecture 4]) conjectured by Kontsevich and Soibelman to construct $\mathcal{D}$ as a homomorphism of algebras and to construct motivic Donaldson-Thomas invariants. In the present paper, we are going to recall some points of the standard language in formal geometry from which the conjecture will be restated in the most precise form (see Conjecture 4.6).

Provided the integral identity conjecture is true the map $\mathcal{D}$ is a homomorphism of $\Gamma$-graded $\mathbb{Q}$-algebras. Consider a certain extension of $\mathcal{D}$ to the completion $\widehat{H}\left(\mathcal{C}_{V}\right)$, for any strict sector $V \subset \mathbb{R}^{2}$. Then the collection of elements $A_{V}^{\text {mot }}=\mathcal{D}\left(A_{V}^{\text {Hall }}\right)$ of the completed motivic quantum tori $\mathcal{R}_{V}$, for all such strict sectors $V$, is called the motivic Donaldson-Thomas invariants of the category $\mathcal{C}$. By construction, the motivic Donaldson-Thomas invariants $A_{V}^{\text {mot }}$ also satisfy the factorization property since the elements $A_{V}^{\text {Hall }}$ do (cf. [17, Theorem 7]).

Because everything behind the integral identity conjecture is motivic integration in the sense of Sebag, Loeser and Nicaise for formal schemes and rigid varieties (cf. [29], [22], [24] and [28], etc.), we shall express in what follows some important points of this theory. On the other hand, it is a fact that Hrushovski-Kazhdan's motivic integration (cf. [13] and [14]) also plays a certain role in completing a proof for the conjecture, we thus devote the last section to mention it in practical aspects.

Acknowledgement. The author sincerely thanks the Basque Centre for Applied Mathematics (BCAM) for hospitality during his visit. 


\section{SPECIAL FORMAL SCHEMES AND ASSOCIATED RIGID VARIETIES}

Throughout the present paper, we work over a non-archimedean complete discretely valued field $K$ of equal characteristics zero, with valuation $\operatorname{ring} R$, with $\mathfrak{m}$ the maximal ideal of $R$, and with residue field $k=R / \mathfrak{m}$. Let us fix a uniformizing parameter $\varpi$ in $R$, i.e., a generator of the principal ideal $\mathfrak{m}$.

2.1. Special formal schemes. A topological $R$-algebra $\mathcal{A}$ is said to be special if $\mathcal{A}$ is a Noetherian adic ring such that, if $\mathcal{J}$ is an ideal of definition of $\mathcal{A}$, the quotient rings $\mathcal{A} / \mathcal{J}^{n}$, for $n \geq 1$, are finitely generated over $R$. By [2], a topological $R$-algebra $\mathcal{A}$ is special if and only if it is topologically $R$-isomorphic to a quotient of the special $R$-algebra $R\left\{T_{1}, \ldots, T_{n}\right\}\left[\left[S_{1}, \ldots, S_{m}\right]\right]$. An adic $R$-algebra $\mathcal{A}$ is topologically finitely generated over $R$ if it is topologically $R$-isomorphic to a quotient algebra of the algebra of restricted power series $R\left\{T_{1}, \ldots, T_{n}\right\}$. Evidently, every topologically finitely generated $R$-algebra is a special $R$-algebra. We refer to [2, Lemma 1.1] for a list of essential properties of $R$-special algebras.

A formal $R$-scheme $\mathfrak{X}$ is said to be special if $\mathfrak{X}$ is a separated Noetherian adic formal scheme and if it is a finite union of affine formal schemes of the form $\operatorname{Spf}(\mathcal{A})$ with $\mathcal{A}$ a special $R$ algebras. A formal $R$-scheme $\mathfrak{X}$ is topologically of finite type if it is a finite union of affine formal schemes of the form $\operatorname{Spf}(\mathcal{A})$ with $\mathcal{A}$ topologically finitely generated $R$-algebras. It is a fact that the category of separated topologically of finite type formal $R$-schemes is a full subcategory of the category of $R$-special formal schemes, and both admit fiber products. On the other hand, a special formal $R$-scheme is separated topologically of finite type over $R$ if it is $R$-adic. If $\mathfrak{X}$ is a special formal $R$-scheme, any formal completion of $\mathfrak{X}$ is a special formal $R$-scheme. Furthermore, by [30], special formal $R$-schemes are excellent.

A morphism $\mathfrak{Y} \rightarrow \mathfrak{X}$ of special formal $R$-schemes is called a morphism of locally finite type if locally it is isomorphic to a morphism of the form $\operatorname{Spf}(\mathcal{B}) \rightarrow \operatorname{Spf}(\mathcal{A})$ with $\mathcal{B}$ topologically finitely generated over $\mathcal{A}$.

The following are some notation which will be useful later. For $\mathfrak{X}$ a Noetherian adic formal scheme, we denote by $\mathfrak{X}_{0}$ the closed subscheme of $\mathfrak{X}$ defined by the largest ideal of definition of $\mathfrak{X}$. Note that $\mathfrak{X}_{0}$ is a reduced Noetherian scheme, that the correspondance $\mathfrak{X} \mapsto \mathfrak{X}_{0}$ is functorial, and that the natural closed immersion $\mathfrak{X}_{0} \rightarrow \mathfrak{X}$ is a homeomorphism. If $\mathfrak{X}$ is a special formal $R$-scheme, $\mathfrak{X}_{0}$ is a separated $k$-scheme of finite type. We shall denote by $\mathfrak{X}_{s}$ the special fiber $\mathfrak{X} \times_{R} k$ of $\mathfrak{X}$. By definition, $\mathfrak{X}_{s}$ is a formal $k$-scheme. If $\mathfrak{X}$ is separated topologically of finite type then $\mathfrak{X}_{s}$ is a separated $k$-scheme of finite type, and $\mathfrak{X}_{0}=\left(\mathfrak{X}_{s}\right)_{\text {red }}$.

2.2. The generic fiber of a special formal scheme. Let $\mathfrak{X}$ be a special formal $R$-scheme. Then one can associate with $\mathfrak{X}$ a rigid $K$-variety denoted by $\mathfrak{X}_{\eta}$ due to [3] or [7], this rigid variety is called the generic fiber of the formal scheme $\mathfrak{X}$. The generic fiber $\mathfrak{X}_{\eta}$ is separated, but in general, not quasi-compact. Furthermore, the correspondance $\mathfrak{X} \mapsto \mathfrak{X}_{\eta}$ is functorial, i.e., it defines a functor from the category of special formal $R$-schemes to the category of separated rigid $K$-varieties. A special formal $R$-scheme $\mathfrak{X}$ is called generically smooth if its generic fiber $\mathfrak{X}_{\eta}$ is smooth over $K$.

Because of working only on affine formal schemes we shall recall the explicit construction of generic fiber in this case, following [7] and [28]. Now assume that $\mathfrak{X}=\operatorname{Spf}(\mathcal{A})$ with $\mathcal{A}$ a special $R$-algebra. Let $\mathcal{J}$ be the largest ideal of definition of $\mathcal{A}$. For any integer $n \geq 1$, we put

$$
\mathcal{A}\left[\varpi^{-1} \mathcal{J}^{n}\right]:=\left\langle\mathcal{A} \cup \varpi^{-1} \mathcal{J}^{n}\right\rangle \subset \mathcal{A} \otimes_{R} K,
$$

i.e., the subalgebra of $\mathcal{A} \otimes_{R} K$ generated by $\mathcal{A}$ and $\varpi^{-1} \mathcal{J}^{n}$. If $\mathcal{B}_{n}$ denotes the $\mathcal{J}$-adic completion of $\mathcal{A}\left[\varpi^{-1} \mathcal{J}^{n}\right]$, then $\mathcal{C}_{n}=\mathcal{B}_{n} \otimes_{R} K$ is an affinoid $R$-algebra. The inclusion $\mathcal{J}^{n+1} \subset \mathcal{J}^{n}$ 
induces a natural morphism of affinoid $R$-algebras $c_{n}: \mathcal{C}_{n+1} \rightarrow \mathcal{C}_{n}$, and in its turn, $c_{n}$ induces an embedding of affinoid $K$-spaces $\operatorname{Spm}\left(\mathcal{C}_{n}\right) \rightarrow \operatorname{Spm}\left(\mathcal{C}_{n+1}\right)$. Then one defines

$$
\mathfrak{X}_{\eta}=\bigcup_{n \geq 1} \operatorname{Spm}\left(\mathcal{C}_{n}\right) \text {. }
$$

There is a specilization map sp : $\mathfrak{X}_{\eta} \rightarrow \mathfrak{X}$ defined as follows. Let $x \in \mathfrak{X}_{\eta}$, and $\mathcal{I} \subset \mathcal{A} \otimes_{R} K$ the maximal ideal in $\mathcal{A} \otimes_{R} K$ corresponding to $x$. Put $\mathcal{I}^{\prime}=\mathcal{I} \cap \mathcal{A} \subset \mathcal{A}$. Then $\operatorname{sp}(x)$ is the unique maximal ideal of $\mathcal{A}$ containing $\varpi$ and $\mathcal{I}^{\prime}$. If $Z$ is a locally closed subscheme of $\mathfrak{X}_{\text {red }}$ then $\operatorname{sp}^{-1}(Z)$ is an open rigid subvariety of $\mathfrak{X}_{\eta}$, which is canonically isomorphic to $(\mathfrak{X} / Z)_{\eta}$, the generic fiber of the formal completion of $\mathfrak{X}$ along $Z$ (cf. [7, Section 7.1]). In particular, if $Z$ is closed, defined by the ideal $\left(g_{1}, \ldots, g_{s}\right)$ of $\mathcal{A}$, then

$$
\operatorname{sp}^{-1}(Z)=\left\{x \in \mathfrak{X}_{\eta}|| g_{i}(x) \mid<1, i=1, \ldots, s\right\} .
$$

The construction of specialization map can be generalized to any special formal $R$-scheme (cf. [7]).

2.3. Resolution of singularities of a special formal scheme. Conrad in [6] introduces the notion of normalization of a special formal scheme, and Nicaise in [28] recalls the definition of irreducibility in formalism. Let $\mathfrak{X}$ be a special formal $R$-scheme, and $\mathfrak{n}: \widetilde{\mathfrak{X}} \rightarrow \mathfrak{X}$ a normalization map (which is a finite morphism of special formal $R$-schemes). Then $\mathfrak{X}$ is called irreducible if the underlying topological space $|\widetilde{\mathfrak{X}}|=\left|(\widetilde{\mathfrak{X}})_{0}\right|$ is connected. For any special formal $R$-scheme $\mathfrak{X}$, we denote by $\widetilde{\mathfrak{X}}_{i}, i=1, \ldots, r$, the (topologically) connected components of $\widetilde{\mathfrak{X}}$, and by $\mathfrak{n}_{i}$ the restriction of $\mathfrak{n}$ to $\widetilde{\mathfrak{X}}_{i}$. Let $\mathfrak{X}_{i}$ denote the reduced closed subscheme of $\mathfrak{X}$ defined by the kernel of the natural morphism $\mathcal{O}_{\mathfrak{X}} \rightarrow\left(\mathfrak{n}_{i}\right)_{*} \mathcal{O}_{\widetilde{\mathfrak{X}}_{i}}$. Then $\mathfrak{X}_{i}, i=1, \ldots, r$, are the irreducible components of $\mathfrak{X}$. In particular, the irreducible components of an affine special formal $R$-scheme $\operatorname{Spf}(\mathcal{A})$ correspond to the minimal prime ideals of $\mathcal{A}$ (see [28, Lemma 2.29]).

Let $\mathfrak{X}$ be a regular special formal $R$-scheme, i.e., $\mathcal{O}_{\mathfrak{X}, x}$ is regular for any $x \in \mathfrak{X}$, and $\mathfrak{E}$ a closed formal subscheme of $\mathfrak{X}$. Recall from [28] that $\mathfrak{E}$ is a strict normal crossings divisor if, for each $x$ in $\mathfrak{X}$, there exists a regular system of local parameters $\left(x_{0}, \ldots, x_{m}\right)$ in $\mathcal{O}_{\mathfrak{X}, x}$, such that, at $x$, the ideal defining $\mathfrak{E}$ is locally generated by $\prod_{j=0}^{m} x_{j}^{N_{j}}$ for some natural $N_{j}, j=0, \ldots, m$, and such that the irreducible components of $\mathfrak{E}$ are regular. By [28, Lemma-Definition 2.36], if $\mathfrak{E}_{i}$ is an irreducible component of $\mathfrak{E}$ which is defined locally at $x$ by the ideal $\left(x_{i}^{m_{i}}\right)$, then the number $m_{i}$ is constant when $x$ varies on $\mathfrak{E}_{i}$. We call the natural number $m_{i}$ the multiplicity of $\mathfrak{E}_{i}$ and denote it by $m\left(\mathfrak{E}_{i}\right)$. Then, if $\mathfrak{E}_{1}, \ldots, \mathfrak{E}_{r}$ are the irreducible components of $\mathfrak{E}$, we can write $\mathfrak{E}$ as a Weil divisor as follows

$$
\mathfrak{E}=\sum_{i=1}^{r} m\left(\mathfrak{E}_{i}\right) \mathfrak{E}_{i} .
$$

Let $\mathfrak{X}$ be a generically smooth, flat special formal $R$-scheme. By definition, a resolution of singularities of $\mathfrak{X}$ is a proper morphism of flat special formal $R$-schemes $\mathfrak{h}: \mathfrak{Y} \rightarrow \mathfrak{X}$, such that $\mathfrak{h}$ induces an isomorphism on the generic fibers, $\mathfrak{Y}$ is regular with the special fiber $\mathfrak{Y}_{s}$ a strict normal crossings divisor. As seen in the following theorem, in the case characreristic zero, such a resolution of singularities of a generically smooth, flat special formal $R$-scheme does exist.

Theorem 2.1 ([30], Theorem 3.4.1, and [28], Proposition 2.43)). Any generically smooth flat special formal $R$-scheme $\mathfrak{X}$ admits a resolution of singularities. If, in addition, $\mathfrak{X}$ is affine, one can get the resolution of singularities by means of formal admissible blow-ups. 
Let us explain some terminologies in the previous theorem, following [28]. Let $\mathfrak{X}$ be a Noetherian adic formal $R$-scheme, $\mathcal{J}$ an ideal of definition of $\mathfrak{X}$, and $\mathcal{I}$ a coherent ideal sheaf on $\mathfrak{X}$. Then by definition, the formal blow-up of $\mathfrak{X}$ with center $\mathcal{I}$ is the morphism of formal schemes

$$
\mathfrak{Y}:=\lim _{n \geq 1} \operatorname{Proj}\left(\bigoplus_{m \geq 0} \mathcal{I}^{m} \otimes \mathcal{O}_{\mathfrak{X}}\left(\mathcal{O}_{\mathfrak{X}} / \mathcal{J}^{n}\right)\right) \rightarrow \mathfrak{X}
$$

Note that $\mathfrak{Y}$ is an adic formal $R$-scheme, and that the ideal $\mathcal{I} \mathcal{O}_{\mathfrak{Y}}$ is invertible on $\mathfrak{Y}$. The blow-up $\mathfrak{h}: \mathfrak{Y} \rightarrow \mathfrak{X}$ satisfies the following universal property: for each morphism of adic formal $R$-schemes $\mathfrak{h}^{\prime}: \mathfrak{Y}^{\prime} \rightarrow \mathfrak{X}$ such that $\mathcal{I O}_{\mathfrak{Y}^{\prime}}$ is invertible, there exists a unique morphism of formal $R$-schemes $\theta: \mathfrak{Y}^{\prime} \rightarrow \mathfrak{Y}$ such that $\mathfrak{h}^{\prime}=\mathfrak{h} \circ \theta$. Furthermore, the formal blow-up $\mathfrak{h}: \mathfrak{Y} \rightarrow \mathfrak{X}$ commutes with flat base change, with the completion of $\mathfrak{X}$ along a closed subscheme $\mathfrak{Z} \subset \mathfrak{X}_{s}$ as well (cf. [28, Proposition 2.16]).

Assume that $\mathfrak{X}$ is a special formal $R$-scheme, and $\mathcal{I}$ is open with respect to the $\varpi$-adic topology, i.e., $\mathcal{I}$ contains a power of $\varpi$. Within this condition, the blow-up $\mathfrak{h}: \mathfrak{Y} \rightarrow \mathfrak{X}$ with center $\mathcal{I}$ is called admissible. By [28, Corollary 2.17], if $\mathfrak{h}: \mathfrak{Y} \rightarrow \mathfrak{X}$ is an admissible blow-up, $\mathfrak{Y}$ is a special formal $R$-scheme, and if, in addition, $\mathfrak{X}$ is $R$-flat, so is $\mathfrak{Y}$. Furthermore, the induced morphism of rigid $K$-varieties $\mathfrak{h}_{\eta}: \mathfrak{Y}_{\eta} \rightarrow \mathfrak{X}_{\eta}$ is an isomorphism [28, Proposition 2.19].

Here is the definition of dilatation of a flat special formal scheme. Let $\mathfrak{X}$ be such a formal $R$-scheme, and $\mathcal{I}$ a coherent ideal sheaf on $\mathfrak{X}$ containing $\varpi$. Let $\mathfrak{h}: \mathfrak{Y} \rightarrow \mathfrak{X}$ be the admissible blow-up with center $\mathcal{I}$. Then, if $\mathfrak{U}$ is the open formal subscheme of $\mathfrak{Y}$ where $\mathcal{I O}_{\mathfrak{Y}}$ is generated by $\varpi$, we call $\mathfrak{U} \rightarrow \mathfrak{X}$ the dilatation of $\mathfrak{X}$ with center $\mathcal{I}$. Like admissible blow-ups, dilatations commute with flat base change, with the formal completion along closed subschemes (cf. [28, Propositions 2.21, 2.23]). Furthermore, by [28, Proposition 2.22], if $\mathcal{I}$ is open, $\mathfrak{U}$ is separated topologically of finite type.

\section{Motivic Integration on Formal $R$-SChemes}

3.1. The Greenberg functor. The main reference for this paragraph is [12]; we may see also [29] and [22].

For $n \geq 0$, let $R_{n}:=R /(\varpi)^{n+1}$. In [12], Greenberg showed that, for any $R_{n}$-scheme $X$ topologically of finite type, the functor $Y \mapsto \operatorname{Hom}_{R_{n}}\left(Y \times_{k} R_{n}, X\right)$ from the category of $k$ schemes to the category of sets is presented by a $k$-scheme $\operatorname{Gr}_{n}(X)$ topologically of finite type such that, for any $k$-algebra $A$,

$$
\operatorname{Gr}_{n}(X)(A)=X\left(A \otimes_{k} R_{n}\right) .
$$

Let $\mathfrak{X}$ be a formal $R$-scheme quasi-compact, separated, topologically of finite type. Then it can be considered as the inductive limit of the $R_{n}$-schemes $X_{n}=\left(\mathfrak{X}, \mathcal{O}_{\mathfrak{X}} \otimes_{R} R_{n}\right)$ in the category of formal $R$-schemes. The canonical truncation morphisms $R_{n+1} \rightarrow R_{n}$ induce canonical morphisms of $k$-schemes

$$
\theta_{n}^{n+1}: \operatorname{Gr}_{n+1}\left(X_{n+1}\right) \rightarrow \operatorname{Gr}_{n}\left(X_{n}\right)
$$

for every integer $n \geq 0$. This follows that there is a canonical way to associate the formal $R$-scheme $\mathfrak{X}$ with a projective system $\left\{\operatorname{Gr}_{n}\left(X_{n}\right)\right\}_{n \in \mathbb{N}}$ in the category of separated $k$-schemes of finite type. The morphisms $\theta_{n}^{n+1}$ being affine, the projective limit $\operatorname{Gr}(\mathfrak{X})$ of the system $\left\{\operatorname{Gr}_{n}\left(X_{n}\right)\right\}_{n \in \mathbb{N}}$ exists in the category of $k$-schemes. Note that one may write also $\operatorname{Gr}_{n}(\mathfrak{X})$ for $\operatorname{Gr}_{n}\left(X_{n}\right)$. The following lemma is useful for Section 3.2.

Lemma 3.1 (Greenberg [12]). The functor Gr respects open and closed immersions and fiber products, and it sends affine topologically of finite type formal $R$-schemes to affine $k$-schemes. 
For a formal $R$-scheme $\mathfrak{X}$ quasi-compact separated topologically of finite type, for $n \in \mathbb{N}$, we denote by $\pi_{n, \mathfrak{X}}$, or simply $\pi_{n}$, the canonical projection $\operatorname{Gr}(\mathfrak{X}) \rightarrow \operatorname{Gr}_{n}\left(X_{n}\right)$. By [29], the image $\pi_{n}(\operatorname{Gr}(\mathfrak{X}))$ of $\operatorname{Gr}(\mathfrak{X})$ in $\operatorname{Gr}_{n}\left(X_{n}\right)$ is a constructible subset of $\operatorname{Gr}_{n}\left(X_{n}\right)$. If, in addition, $\mathfrak{X}$ is smooth and of relative dimension $d$, then by [29, Lemma 3.4.2],

$\star$ the morphism $\pi_{n}: \operatorname{Gr}(\mathfrak{X}) \rightarrow \operatorname{Gr}_{n}\left(X_{n}\right)$ is surjective, and

* the canonical projection $\operatorname{Gr}_{n+m}\left(X_{n+m}\right) \rightarrow \operatorname{Gr}_{n}\left(X_{n}\right)$ is a locally trivial fibration for the Zariski topology with fiber $\mathbb{A}_{k}^{d m}$.

We refer to [29, Section 4.2] for the definition of piecewise trivial fibration mentioned in the following

Proposition 3.2 (Sebag [29], Lemma 4.3.25). Let $\mathfrak{X}$ be a flat separated, quasi-compact, topologically of finite type formal $R$-scheme of relative dimension $d$. There is an integer $c \geq 1$ such that, for $e \in \mathbb{Z}$ and $n \in \mathbb{N}$ with $n \geq c e$, the projection

$$
\pi_{n+1}(\operatorname{Gr}(\mathfrak{X})) \rightarrow \pi_{n}(\operatorname{Gr}(\mathfrak{X}))
$$

is a piecewise trivial fibration over $\pi_{n}\left(\mathrm{Gr}^{(e)}(\mathfrak{X})\right)$ with fiber $\mathbb{A}_{k}^{d}$, where

$$
\operatorname{Gr}^{(e)}(\mathfrak{X})=\operatorname{Gr}(\mathfrak{X}) \backslash \pi_{e}^{-1}\left(\operatorname{Gr}_{e}\left(\mathfrak{X}_{\text {sing,e }}\right)\right) .
$$

3.2. Loeser-Sebag's motivic integration. We have mentioned the Grothendieck ring $\mathcal{M}_{k}^{\hat{\mu}}$ from beginning, but it is not sufficient in the framework of motivic integration of Sebag, Loeser and Nicaise. Most preferably, we refer to [10] and [25] for a useful relative version of it. Let $X$ be an algebraic $k$-variety, viewed as acted trivially by $\hat{\mu}$, and let $\operatorname{Var}_{X, \hat{\mu}}$ be the category of $X$-varieties endowed with good $\hat{\mu}$-action. By definition, a good $\hat{\mu}$-action on an $X$-variety $Y$ is a group action $\mu_{n} \times Y \rightarrow Y$ for some $n \in \mathbb{N}_{>0}$, which is a morphism of $X$-varieties, such that each orbit is contained in an affine $k$-subvariety of $Y$. The Grothendieck group $K_{0}\left(\operatorname{Var}_{X, \hat{\mu}}\right)$ is an abelian group generated by the $\hat{\mu}$-equivariant isomorphism classes $[Y \rightarrow X, \sigma]$ modulo the conditions

$$
[Y \rightarrow X, \sigma]=\left[V \rightarrow X,\left.\sigma\right|_{V}\right]+\left[Y \backslash V \rightarrow X,\left.\sigma\right|_{Y \backslash V}\right]
$$

for any Zariski closed subvariety $V$ of $Y$, and

$$
\left[Y \times \mathbb{A}_{k}^{n} \rightarrow X, \sigma\right]=\left[Y \times \mathbb{A}_{k}^{n} \rightarrow X, \sigma^{\prime}\right]
$$

if $\sigma$ and $\sigma^{\prime}$ lift the same $\hat{\mu}$-action on $Y \rightarrow X$ to an affine action on $Y \times \mathbb{A}_{k}^{n} \rightarrow X$. Using fiber product of $X$-varieties, one may equip $K_{0}\left(\operatorname{Var}_{X, \hat{\mu}}\right)$ with a natural structure of ring. We also write $\mathbb{L}$ for the class $\left[\mathbb{A}_{k}^{1} \times X \rightarrow X\right.$, trivial $\hat{\mu}$-action], and define

$$
\mathcal{M}_{X}^{\hat{\mu}}:=K_{0}\left(\operatorname{Var}_{X, \hat{\mu}}\right)\left[\mathbb{L}^{-1}\right] \text { and } \mathcal{M}_{X, \mathrm{loc}}^{\hat{\mu}}:=\mathcal{M}_{X}^{\hat{\mu}}\left[\left\{\left(1-\mathbb{L}^{i}\right)^{-1}\right\}_{i \in \mathbb{N}}\right] .
$$

For simplicity, we consider an element of $\mathcal{M}_{X}^{\hat{\mu}}$ as an element of $\mathcal{M}_{X \text {,loc }}^{\hat{\mu}}$. Any morphism of $k$-varieties $f: X \rightarrow Y$ induces a morphism of groups $f_{!}: \mathcal{M}_{X}^{\hat{\mu}} \rightarrow \mathcal{M}_{Y}^{\hat{\mu}}$ by composition, and a morphism of rings $f^{*}: \mathcal{M}_{Y}^{\hat{\mu}} \rightarrow \mathcal{M}_{X}^{\hat{\mu}}$ by fiber product. We write simply $\int_{X}$ for $(X \rightarrow \operatorname{Spec}(k))_{!}$. Forgetting the action we obtain Grothendieck rings, which are denoted by $\mathcal{M}_{X}$ and $\mathcal{M}_{X \text {,loc }}$.

The previous definition of Grothendieck rings still makes sense for schemes of finite type and morphisms between them. This is important since in this paper we also need to work with base $X$ being a $k$-scheme of finite type (e.g., the reduction of a special formal scheme). By abuse of notation we shall also denote these Grothendieck rings of schemes of finite type by $\mathcal{M}_{X}, \mathcal{M}_{X, \text { loc }}, \mathcal{M}_{X}^{\hat{\mu}}$ and $\mathcal{M}_{X, \text { loc }}^{\hat{\mu}}$.

Let $\mathfrak{X}$ be a formal $R$-scheme topologically of finite type. By definition, a subset $A$ of $\operatorname{Gr}(\mathfrak{X})$ is cylindrical of level $n \geq 0$ if $A=\pi_{n}^{-1}(C)$ with $C$ a constructible subset of $\operatorname{Gr}_{n}(\mathfrak{X})$. Denote by 
$\mathbf{C}_{\mathfrak{X}}$ the set of cylindrical subsets of $\operatorname{Gr}(\mathfrak{X})$ of some level. Then $\mathbf{C}_{\mathfrak{X}}$ is a Boolean algebra, and it is stable by finite intersection, finite union and by taking complements. If $A$ is cylindrical of some level, then $\pi_{n}(A)$ is constructible for any $n \geq 0$ (cf. [22]).

Assume in addition that $\mathfrak{X}$ is flat and of relative dimension $d$. A cylinder $A$ of $\operatorname{Gr}(\mathfrak{X})$ is called stable of level $n$ if it is cylindrical of level $n$, and if for every $m \geq n$ the morphism $\pi_{m+1}(\operatorname{Gr}(\mathfrak{X})) \rightarrow \pi_{m}(\operatorname{Gr}(\mathfrak{X}))$ is a piecewise trivial fibration over $\pi(A)$ with fiber $\mathbb{A}_{k}^{d}$. Note that if $\mathfrak{X}$ is smooth, every cylinder in $\operatorname{Gr}(\mathfrak{X})$ is stable. We shall denote by $\mathbf{C}_{0, \mathfrak{X}}$ the set of stable cylindrical subsets of $\operatorname{Gr}(\mathfrak{X})$ of some level. In general $\mathbf{C}_{0, \mathfrak{X}}$ is not a Boolean algebra, but it is an ideal of $\mathbf{C}_{\mathfrak{X}}$.

Proposition 3.3 (Lê [19], Proposition 5.1). There exists a unique additive morphism

$$
\widetilde{\mu}: \mathbf{C}_{0, \mathfrak{X}} \rightarrow \mathcal{M}_{\mathfrak{X}_{0}}
$$

such that $\widetilde{\mu}(A)=\left[\pi_{n}(A)\right] \mathbb{L}^{-(n+1) d}$ for $A$ a stable cylinder of level $n$.

Let $\widehat{\mathcal{M}}_{\mathfrak{X}_{0}}$ denote the completion of $\mathcal{M}_{\mathfrak{X}_{0}}$ with respect to a filtration $F^{\bullet} m$-piece of which $F^{m} \mathcal{M}_{\mathfrak{X}_{0}}$ is the subgroup of $\mathcal{M}_{\mathfrak{X}_{0}}$ generated by $[S] \mathbb{L}^{-i}$ with $\operatorname{dim}(S)-i \leq-m$. As similarly explained in $[22]^{3}$, one can extend $\widetilde{\mu}$ to a unique additive morphism $\mu: \mathbf{C}_{\mathfrak{X}} \rightarrow \widehat{\mathcal{M}}_{\mathfrak{X}_{0}}$ with the property that

$$
\mu(A)=\lim _{e \rightarrow \infty} \widetilde{\mu}\left(A \cap \mathrm{Gr}^{(e)}(\mathfrak{X})\right),
$$

where the limit on the right hand side exists in $\widehat{\mathcal{M}}_{\mathfrak{X}_{0}}$ according to [22, Proposition 3.6.2]. Furthermore, consider a larger class $\mathbf{D}_{\mathfrak{X}}$ containing $\mathbf{C}_{\mathfrak{X}}$ of measurable subsets of $\mathrm{Gr}(\mathfrak{X})$. By a measurable subset $A$ of $\operatorname{Gr}(\mathfrak{X})$ we mean for every positive real number $\epsilon$ there exists a sequence of cylindrical subsets $A_{i}(\epsilon)(i \in \mathbb{N})$ with the symmetric difference $A \cup A_{0}(\epsilon) \backslash A \cap A_{0}(\epsilon)$ contained in $\bigcup_{i \geq 1} A_{i}(\epsilon)$ and $\left\|\mu\left(A_{i}(\epsilon)\right)\right\| \leq \epsilon$ for all $i$ in $\mathbb{N}_{>0}$. Then, once again, $\mu$ can be extended to a unique additive morphism $\mu: \mathbf{D}_{\mathfrak{X}} \rightarrow \widehat{\mathcal{M}}_{\mathfrak{X}_{0}}$ such that

$$
\mu(A)=\lim _{\epsilon \rightarrow 0} \mu\left(A_{0}(\epsilon)\right)
$$

for any sequence $A_{i}(\epsilon)$ in the definition of measurability of the set $A$ (see Definition 3.7.1 and Theorem 3.7.2 of [22]).

Definition 3.4. For a measurable subset $A$ of $\operatorname{Gr}(\mathfrak{X})$ and a function $\alpha: A \rightarrow \mathbb{Z} \cup\{\infty\}$, we say that $\mathbb{L}^{-\alpha}$ is integrable or that $\alpha$ is exponential integrable if the fibers of $\alpha$ are measurable and if the following sum (motivic integral) converges in $\widehat{\mathcal{M}}_{\mathfrak{X}_{0}}$

$$
\int_{A} \mathbb{L}^{-\alpha} d \mu:=\sum_{n \in \mathbb{Z}} \mu\left(\alpha^{-1}(n)\right) \mathbb{L}^{-n} .
$$

If all the fibers $\alpha^{-1}(n)$ are stable cylinders and $\alpha$ takes only a finite number of values on $A$, we can use the restriction $\widetilde{\mu}$ instead of $\mu$, and the motivic integral then takes values in $\mathcal{M}_{\mathfrak{X}_{0}}$. In this case, we shall denote the integral by $\int_{A} \mathbb{L}^{-\alpha} d \widetilde{\mu}$.

3.3. Integral of a differential form. Let $\mathfrak{X}$ be a flat quasi-compact separated topologically of finite type generically smooth formal $R$-scheme of relative dimension $d$, and let $\omega$ be a differential form in $\Omega_{\mathfrak{X} \mid R}^{d}(\mathfrak{X})$. Let $x$ be a point of $\operatorname{Gr}(\mathfrak{X}) \backslash \operatorname{Gr}\left(\mathfrak{X}_{\text {sing }}\right)$ defined over some field extension $k^{\prime}$ of $k$. Let $R^{\prime}=R \widehat{\otimes}_{k} k^{\prime}$, and let $\varphi: \operatorname{Spf}\left(R^{\prime}\right) \rightarrow \mathfrak{X}$ be the morphism of formal $R$-schemes corresponding to $x$. Since $L:=\left(\varphi^{*} \Omega_{\mathfrak{X} \mid R}^{d}\right) /($ torsion $)$ is a free $\mathcal{O}_{R^{\prime}}$-module of rank 1 ,

\footnotetext{
${ }^{3} \operatorname{In}[22]$, the measure $\widetilde{\mu}$ is defined to take values in the ring $\mathcal{M}_{k}$.
} 
its submodule $M$ generated by $\varphi^{*} \omega$ is either zero or $\varpi^{n} \mathrm{~L}$ for some $n \in \mathbb{N}$. Then $\operatorname{ord}_{\varpi}(\omega)(x)$ is defined to be $\infty$ or $n$, respectively.

Because of a canonical isomorphism $\Omega_{\mathfrak{X}_{\eta}}^{d}\left(\mathfrak{X}_{\eta}\right) \cong \Omega_{\mathfrak{X} \mid R}^{d}(\mathfrak{X}) \otimes_{R} K$, one can write a differential form $\omega$ in $\Omega_{\mathfrak{X}_{\eta}}^{d}\left(\mathfrak{X}_{\eta}\right)$ as a product $\omega=\varpi^{-n} \widetilde{\omega}$ with $\widetilde{\omega} \in \Omega_{\mathfrak{X} \mid R}^{d}(\mathfrak{X})$ and $n \in \mathbb{N}$. Then we set $\operatorname{ord}_{\varpi, \mathfrak{X}}(\omega):=\operatorname{ord}_{\varpi}(\widetilde{\omega})-n$. This definition is independent of the choice of $\widetilde{\omega}$ (cf. [28]). Let $\omega$ be a differential form in $\Omega_{\mathfrak{X}_{\eta}}^{d}\left(\mathfrak{X}_{\eta}\right)$. By [22, Theorem-Definition 4.1.2], the function $\operatorname{ord}_{\varpi, \mathfrak{X}}(\omega)$ is exponentially integrable on $\operatorname{Gr}(\mathfrak{X})$, so we can define

$$
\int_{\mathfrak{X}}|\omega|:=\int_{\operatorname{Gr}(\mathfrak{X})} \mathbb{L}^{-\operatorname{ord}_{\varpi, \mathfrak{X}}(\omega)} d \mu \in \widehat{\mathcal{M}}_{\mathfrak{X}_{0}} .
$$

Following [24], by a weak formal Néron model of $\mathfrak{X}_{\eta}$ we mean a smooth formal $R$-scheme $\mathfrak{Y}$ topologically of finite type such that $\mathfrak{Y}_{\eta}$ is an open rigid subspace of $\mathfrak{X}_{\eta}$ and the canonical maps $\mathfrak{Y}(\widetilde{R}) \rightarrow \mathfrak{X}_{\eta}(\widetilde{K})$ are bijective for any finite unramified extension $\widetilde{K}$ of $K$, where $\widetilde{R}$ is the normalization of $R$ in $\widetilde{K}$. By [22, Proposition 2.7.3] there exists a formal model $\mathfrak{X}$ of $\mathfrak{X}_{\eta}$ whose $R$-smooth locus is a weak formal Néron model of $\mathfrak{X}_{\eta}$. Let $\omega$ be a gauge form on $\mathfrak{X}_{\eta}$. Since $\Omega_{\mathfrak{X}_{\eta}}^{d}\left(\mathfrak{X}_{\eta}\right) \cong \Omega_{\mathfrak{X} \mid R}^{d}(\mathfrak{X}) \otimes_{R} K$, one can write $\omega=\varpi^{-n} \widetilde{\omega}$ with $\widetilde{\omega} \in \Omega_{\mathfrak{X} \mid R}^{d}(\mathfrak{X})$ and $n \in \mathbb{N}$, and we put $\operatorname{ord}_{\varpi, \mathfrak{X}}(\omega):=\operatorname{ord}_{\varpi}(\widetilde{\omega})-n$, where this definition is independent of the choice of $\widetilde{\omega}$ due to [22, Section 4.1]. Assume that some open dense formal subscheme $\mathfrak{Y}$ of $\mathfrak{X}$ is a weak formal Néron model of $\mathfrak{X}_{\eta}$. Then, since $\mathfrak{Y}$ is smooth, $\Omega_{\mathfrak{Y} \mid R}^{d}$ is locally free of rank 1 over $\mathcal{O}_{\mathfrak{Y}}$, i.e., there is a open covering $\left\{\mathfrak{U}_{i}\right\}$ of $\mathfrak{Y}$ such that $\Omega_{\mathfrak{Y} \mid R}^{d}\left(\mathfrak{U}_{i}\right)$ is free of rank 1. Therefore, for every $i$, there is an $f_{i}$ in $\mathcal{O}_{\mathfrak{Y}}\left(\mathfrak{U}_{i}\right)$ such that

$$
\widetilde{\omega} \mathcal{O}_{\mathfrak{Y}}\left(\mathfrak{U}_{i}\right) \otimes\left(\Omega_{\mathfrak{Y} \mid R}^{d}(\mathfrak{Y})\right)^{-1} \cong\left(f_{i}\right) \mathcal{O}_{\mathfrak{Y}}\left(\mathfrak{U}_{i}\right) .
$$

It implies that the restriction of the function $\operatorname{ord}_{\varpi}(\widetilde{\omega})$ to $\mathfrak{U}_{i}$ is equal to the function $\operatorname{ord}_{\varpi}\left(f_{i}\right)$ which assigns $\operatorname{ord}_{\varpi}\left(f_{i}(\varphi)\right)$ to a point $\varphi \in \operatorname{Gr}\left(\mathfrak{U}_{i}\right)$. Let $f$ be the global section in $\mathcal{O}_{\mathfrak{Y}}(\mathfrak{U})$ such that $f=f_{i}$ on $\mathfrak{U}_{i}$. Then, by glueing $\operatorname{ord}_{\varpi}\left(f_{i}\right)$ 's altogether, we obtain a function $\operatorname{ord}_{\varpi}(f)$ : $\operatorname{Gr}(\mathfrak{X})=\operatorname{Gr}(\mathfrak{Y}) \rightarrow \mathbb{Z}$ which is equal to $\operatorname{ord}_{\varpi}(\widetilde{\omega})$. Since $\omega$ is a gauge form, $f$ induces an invertible function on $\mathfrak{X}_{\eta}$, hence by the maximum principle ord $\mathfrak{d}_{\varpi}(f)$ takes only a finite number of values (see the proof of Theorem-Definition 4.1.2 of [22]). Therefore $\operatorname{ord}_{\varpi, \mathfrak{X}}(\omega)$ takes only a finite number of values and its fibers are stable cylinders. In this case, we put

$$
\int_{\mathfrak{X}}|\omega|:=\int_{\operatorname{Gr}(\mathfrak{X})} \mathbb{L}^{-\operatorname{ord}_{\varpi, \mathfrak{X}}(\omega)} d \widetilde{\mu} \in \mathcal{M}_{\mathfrak{X}_{0}} .
$$

Note that this definition does not depend on the choice of the Néron model (cf. [28]).

3.4. Motivic integration on special formal schemes. Working on special formal schemes we shall use a stronger notion than weak Néron model, it will be called Néron smoothening. Let $\mathfrak{X}$ be a special formal $R$-scheme. By a Néron smoothening for $\mathfrak{X}$ we mean a morphism of special formal $R$-schemes $\mathfrak{Y} \rightarrow \mathfrak{X}$, with $\mathfrak{Y}$ adic smooth over $R$, which induces an open embedding $\mathfrak{Y}_{\eta} \rightarrow \mathfrak{X}_{\eta}$ satisfying $\mathfrak{Y}_{\eta}(\widetilde{K})=\mathfrak{X}_{\eta}(\widetilde{K})$ for any finite unramified extension $\widetilde{K}$ of $K$.

Proposition 3.5 (Nicaise [28]). Any generically smooth special formal $R$-schemes $\mathfrak{X}$ admits a Néron smoothening $\mathfrak{Y} \rightarrow \mathfrak{X}$. Moreover, we can choose $\mathfrak{Y}$ to be a quasi-compact separated topologically of finite type generically smooth formal $R$-scheme.

In particular, if in addition $\mathfrak{X}$ is flat and $h: \mathfrak{Y}^{\prime} \rightarrow \mathfrak{X}$ is the dilatation with center $\mathfrak{X}_{s}$, then $\mathfrak{Y}^{\prime}$ is a separated topologically of finite type generically smooth formal $R$-scheme (cf. [28]). Hence we can choose a Néron smoothening $\mathfrak{Y}$ for $\mathfrak{X}$ to be a Néron smoothening for $\mathfrak{Y}^{\prime}$. 
In [28], Nicaise defines motivic integral on special formal $R$-schemes in the following way. Let $\mathfrak{X}$ be a flat generically smooth special formal $R$-scheme, and $h: \mathfrak{Y} \rightarrow \mathfrak{X}$ be the dilatation with center $\mathfrak{X}_{s}$. If $\omega$ be a differential form of maximal degree (resp. a gauge form) on $\mathfrak{X}_{\eta}$, then one defines

$$
\int_{\mathfrak{X}}|\omega|:=\int_{\mathfrak{Y}}|\omega| \text { in } \widehat{\mathcal{M}}_{\mathfrak{X}_{0}}\left(\text { resp. in } \mathcal{M}_{\mathfrak{X}_{0}}\right)
$$

(the integrals on the right were already defined in Subsection 3.3). If $\mathfrak{X}$ be a generically smooth special formal $R$-scheme, we denote by $\mathfrak{X}^{\text {flat }}$ its maximal flat closed subscheme (obtained by killing $\varpi$-torsion), and define

$$
\int_{\mathfrak{X}}|\omega|:=\int_{\mathfrak{X}^{\text {flat }}}|\omega| \text {. }
$$

The following proposition gives an equivalent definition of integral on special formal $R$ schemes $\mathfrak{X}$ using a Néron smoothening for $\mathfrak{X}$.

Proposition 3.6 (Nicaise [28], Propositions 4.7, 4.8). Let $\mathfrak{X}$ be a generically smooth special formal $R$-scheme, and $\mathfrak{Y} \rightarrow \mathfrak{X}$ a Néron smoothening for $\mathfrak{X}$. If $\omega$ is a differential form of maximal degree (resp. a gauge form) on $\mathfrak{X}_{\eta}$, then

(i) the identity $\int_{\mathfrak{X}}|\omega|=\int_{\mathfrak{Y}}|\omega|$ holds in $\widehat{\mathcal{M}}_{\mathfrak{X}_{0}}$ (resp. in $\mathcal{M}_{\mathfrak{X}_{0}}$ );

(ii) the image of $\int_{\mathfrak{X}}|\omega|$ under the forgetful $\widehat{\mathcal{M}}_{\mathfrak{X}_{0}} \rightarrow \widehat{\mathcal{M}}_{k}$ (resp. $\mathcal{M}_{\mathfrak{X}_{0}} \rightarrow \mathcal{M}_{k}$ ) only depends on $\mathfrak{X}_{\eta}$, not on $\mathfrak{X}$. We shall denote it by $\int_{\mathfrak{X}_{\eta}}|\omega|$.

3.5. Motivic integration on smooth rigid varieties. Let $\mathfrak{X}$ be a generically smooth special formal $R$-scheme. We consider the forgetful morphisms

$$
\widehat{\int_{\mathfrak{X}_{0}}}: \widehat{\mathcal{M}}_{\mathfrak{X}_{0}} \rightarrow \widehat{\mathcal{M}}_{k} \text { and } \int_{\mathfrak{X}_{0}}: \mathcal{M}_{\mathfrak{X}_{0}} \rightarrow \mathcal{M}_{k} .
$$

By Proposition 3.6, if $\omega$ is a differential form in $\Omega_{\mathfrak{X}_{\eta}}^{d}\left(\mathfrak{X}_{\eta}\right)$, then $\widehat{\int_{\mathfrak{X}_{0}}}\left(\int_{\mathfrak{X}}|\omega|\right)$ in $\widehat{\mathcal{M}}_{k}$ only depends on $\mathfrak{X}_{\eta}$, not on $\mathfrak{X}$ (cf. [22, Proposition 4.2.1]). One may define motivic integral on such type of rigid $K$-varieties as follows

$$
\int_{\mathfrak{X}_{\eta}}|\omega|:=\int_{\mathfrak{X}_{0}}\left(\int_{\mathfrak{X}}|\omega|\right) \in \widehat{\mathcal{M}}_{k} .
$$

If $\omega$ is a gauge form on $\mathfrak{X}_{\eta}$, the image $\int_{\mathfrak{X}_{0}}\left(\int_{\mathfrak{X}}|\omega|\right)$ in $\mathcal{M}_{k}$ depend only on $\mathfrak{X}_{\eta}$, thus we put

$$
\int_{\mathfrak{X}_{\eta}}|\omega|:=\int_{\mathfrak{X}_{0}}\left(\int_{\mathfrak{X}}|\omega|\right) \in \mathcal{M}_{k} .
$$

(See more in [22, Section 4].)

The previous type of rigid $K$-varieties (i.e., the generic fiber of special formal $R$-schemes) is in fact a particular case of bounded rigid varieties according to Nicaise-Sebag [26]. A rigid $K$-variety $X$ is bounded if there exists a quasi-compact open subspace $Y$ of $X$ such that $Y(\widetilde{K})=X(\tilde{K})$ for any finite unramified extension $\widetilde{K}$ of $K$. On a quasi-compact smooth rigid variety the motivic integral was already defined by Loeser-Sebag [22], and inspired by this, Nicaise-Sebag [26] extend the notion to bounded smooth rigid varieties. If the previous $X$ is smooth, so is $Y$, and one can define

$$
\int_{X}|\omega|:=\int_{Y}|\omega|
$$


In general, $\int_{X}|\omega|$ lives in $\widehat{\mathcal{M}}_{k}$. However, if $\omega$ is a gauge form, then $\int_{X}|\omega|$ belongs to $\mathcal{M}_{k}$. The integral is well defined, due to [26, Proposition 5.9].

For any integer $d \geq 0$, let $\operatorname{GBSRig}_{K}^{d}$ be the category of gauged bounded smooth rigid $K$ varieties of dimension $d$, in which an object of $\operatorname{GBSRig}_{K}^{d}$ is a pair $(X, \omega)$ with $X$ a bounded smooth rigid $K$-variety of dimension $d$ and $\omega$ a gauge form on $X$, and a morphism $h$ : $\left(X^{\prime}, \omega^{\prime}\right) \rightarrow(X, \omega)$ in GBSRig $_{K}^{d}$ is a morphism of bounded smooth rigid $K$-varieties $h: X^{\prime} \rightarrow X$ such that $h^{*} \omega=\omega^{\prime}$. The Grothendieck group $K\left(\operatorname{GBSRig}_{K}^{d}\right)$ is the quotient of the free abelian group generated by symbols $[X, \omega]$ with $(X, \omega)$ in $\operatorname{ObGBSRig}_{K}^{d}$ by the relation $\left[X^{\prime}, \omega^{\prime}\right]=[X, \omega]$ if $\left(X^{\prime}, \omega^{\prime}\right) \cong(X, \omega)$ in $\operatorname{GBSRig}_{K}^{d}$, and

$$
[X, \omega]=\sum_{\emptyset \neq I \subset J}(-1)^{|I|-1}\left[O_{I},\left.\omega\right|_{O_{I}}\right],
$$

whenever $\left(O_{i}\right)_{i \in J}$ is a finite admissible covering of $X, O_{I}=\bigcap_{i \in I} O_{i}$ for any $I \subset J$. We put

$$
K\left(\operatorname{GBSRig}_{K}\right):=\bigoplus_{d \geq 0} K\left(\operatorname{GBSRig}_{K}^{d}\right)
$$

and defines a product on it as follows

$$
[X, \omega] \cdot\left[X^{\prime}, \omega^{\prime}\right]:=\left[X \times X^{\prime}, \omega \times \omega^{\prime}\right] .
$$

Equipped with this product the Grothendieck group $K\left(\operatorname{GBSRig}_{K}\right)$ becomes a ring.

Proposition 3.7 (Lê [19], Proposition 5.3). There exists a unique homomorphism of rings $\Phi: K\left(\operatorname{GBSRig}_{K}\right) \rightarrow \mathcal{M}_{k}$ such that

$$
\Phi([X, \omega])=\int_{X}|\omega|
$$

\section{Motivic NeARby CyCles And the integral IDEntity CONJECture}

4.1. Motivic volume. For $m \in \mathbb{N}_{>0}$, let $K(m):=K[T] /\left(T^{m}-\varpi\right), R(m):=R[T] /\left(T^{m}-\varpi\right)$. For any formal $R$-scheme $\mathfrak{X}$, we define $\mathfrak{X}(m):=\mathfrak{X} \times_{R} R(m)$ and $\mathfrak{X}_{\eta}(m):=\mathfrak{X}_{\eta} \times_{K} K(m)$. If $\omega$ is a differential form on $\mathfrak{X}_{\eta}$, we denote by $\omega(m)$ the pullback of $\omega$ via the natural morphism $\mathfrak{X}_{\eta}(m) \rightarrow \mathfrak{X}_{\eta}$.

Let $\mathfrak{X}$ be a generically smooth special formal $R$-scheme, $\omega$ a gauge form on $\mathfrak{X}_{\eta}$. According to [28], the volume Poincaré series of $(\mathfrak{X}, \omega)$ is defined to be an element of $\mathcal{M}_{\mathfrak{X}_{0}}[[T]]$ as

$$
S(\mathfrak{X}, \omega ; T):=\sum_{m \geq 1}\left(\int_{\mathfrak{X}(m)}|\omega(m)|\right) T^{m} .
$$

We remark that volume Poincaré series in motivic integration was firstly introduced by Nicaise and Sebag in [24] for any generically smooth separated formal $R$-scheme topologically of finite type together with a gauge form on its generic fiber. With the previous definition we recall a generalization of the volume Poincaré series by Nicaise in the framework of motivic integration for special formal schemes (see [28]).

In general, by [28, Remark 4.10], the volume Poincaré series $S(\mathfrak{X}, \omega ; T)$ depends on the choice of $\varpi$, i.e., on the $K$-fields $K(m)$. However, if $k$ is an algebraically closed field, $K(m)$ is the unique extension of degree $m$ of $K$, up to $K$-isomorphism, hence $S(\mathfrak{X}, \omega ; T)$ is independent of the choice of $\varpi$. In [19], we endow $\int_{\mathfrak{X}(m)}|\omega(m)|$ with $\mu_{m}$-action for every $m \in \mathbb{N}_{>0}$ so that $S(\mathfrak{X}, \omega ; T)$ lives well in $\mathcal{M}_{\mathfrak{X}_{0}}^{\hat{\mu}}[[T]]$. 
By Theorem 2.1, there exists a resolution of singularities of $\mathfrak{X}$. Fix such a resolution of singularities $\mathfrak{h}: \mathfrak{Y} \rightarrow \mathfrak{X}$. Let $\mathfrak{E}_{i}, i \in J$, be the irreducible components of divisor $\mathfrak{Y}_{s}=\mathfrak{h}^{-1}\left(\mathfrak{X}_{s}\right)$. Assume that the divisor $\mathfrak{Y}_{s}$ is written as $\mathfrak{Y}_{s}=\sum_{i \in J} N_{i} \mathfrak{E}_{i}$. For nonempty $I \subset J$, we put

$$
\mathfrak{E}_{I}=\bigcap_{i \in I} \mathfrak{E}_{i}, \quad \mathfrak{E}_{I}^{\circ}=\mathfrak{E}_{I} \backslash \bigcup_{j \notin I} \mathfrak{E}_{j}
$$

Let $E_{i}=\left(\mathfrak{E}_{i}\right)_{0}, i \in J$, and $E_{I}=\bigcap_{i \in I} E_{i}, E_{I}^{\circ}=E_{I} \backslash \bigcup_{j \notin I} E_{j}, \emptyset \neq I \subset J$. Then $E_{I}=\left(\mathfrak{E}_{I}\right)_{0}$ for any $\emptyset \neq I \subset J$. In particular, if $\mathfrak{X}$ is separated topologically of finite type, $E_{i}=\mathfrak{E}_{i}$, for any $i \in J$.

Let $U$ be an affine Zariski open subset of $\mathfrak{Y}_{0}$ such that $U \cap E_{I}^{\circ} \neq \emptyset$, and on $U \cap E_{I}^{\circ}$, $\mathfrak{f}_{0} \circ \mathfrak{h}_{0}=\widetilde{u} \prod_{i \in I} y_{i}^{N_{i}}$, with $\widetilde{u}$ a unit and $y_{i}$ a local coordinate defining $E_{i}$. Let $m_{I}$ denote the greatest common divisor of $\left(N_{i}\right)_{i \in I}$. Define an unramified Galois covering $\pi_{I}: \widetilde{E}_{I}^{\circ} \rightarrow E_{I}^{\circ}$ with Galois group $\mu_{m_{I}}$ given over $U \cap E_{I}^{\circ}$ by

$$
U \cap \widetilde{E}_{I}^{\circ}:=\left\{(z, y) \in \mathbb{A}_{k}^{1} \times\left(U \cap E_{I}^{\circ}\right) \mid z^{m_{I}}=\widetilde{u}(y)^{-1}\right\} .
$$

Choose a covering of $E_{I}^{\circ}$ consisting of such open subsets $U \cap E_{I}^{\circ}$, then the Galois coverings $U \cap \widetilde{E}_{I}^{\circ}$ over $U \cap E_{I}^{\circ}$ can be then glued together in an obvious way to a covering $\widetilde{E}_{I}^{\circ}$, which has a natural $\mu_{m_{I}}$-action (obtained by multiplying the $z$-coordinate with elements of $\mu_{m_{I}}$ ). The $\mu_{m_{I}}$-action induces a good $\hat{\mu}$-action over $E_{I}^{\circ}$ (cf. [8], [10] and [4]). Then the $\hat{\mu}$-equivariant morphism $\mathfrak{h}_{0} \circ \pi_{I}: \widetilde{E}_{I}^{\circ} \rightarrow \mathfrak{X}_{0}$ defines a class $\left[\widetilde{E}_{I}^{\circ}\right]$ in $\mathcal{M}_{\mathfrak{X}_{0}}^{\hat{\mu}}$.

The following is a generalization of Nicaise [28, Corollary 7.13] to the version with action.

Theorem 4.1. Let $\mathfrak{X}$ be a generically smooth special formal $R$-scheme of relative dimension d. Let $\mathfrak{Y} \rightarrow \mathfrak{X}$ be a resolution of singularities with special fiber $\mathfrak{Y}_{s}=\sum_{i \in J} N_{i} \mathfrak{E}_{i}$. Let $\omega$ be an $\mathfrak{X}$-bounded gauge form on $\mathfrak{X}_{\eta}$ and $\mu_{i}=\operatorname{ord}_{\mathfrak{E}_{i}}(\omega)$ for $i \in J$. Then the following identity

$$
S(\mathfrak{X}, \omega ; T)=\mathbb{L}^{-d} \sum_{\emptyset \neq I \subset J}(\mathbb{L}-1)^{|I|-1}\left[\widetilde{E}_{I}^{\circ}\right] \prod_{i \in I} \frac{\mathbb{L}^{-\mu_{i}} T^{N_{i}}}{1-\mathbb{L}^{-\mu_{i}} T^{N_{i}}}
$$

in $\mathcal{M}_{\mathfrak{X}_{0}}^{\hat{\mu}}[[T]]$.

The limit $S\left(\mathfrak{X}, \widehat{K^{s}}\right):=-\lim _{T \rightarrow \infty} S(\mathfrak{X}, \omega ; T)$ is independent of the choice of $\omega$, namely

$$
S\left(\mathfrak{X}, \widehat{K^{s}}\right)=\mathbb{L}^{-d} \sum_{\emptyset \neq I \subset J}(1-\mathbb{L})^{|I|-1}\left[\widetilde{E}_{I}^{\circ}\right] \in \mathcal{M}_{\mathfrak{X}_{0}}^{\hat{\mu}},
$$

and called motivic volume of $\mathfrak{X}$. As shown in [27], in fact, $S\left(\mathfrak{X}, \widehat{K^{s}}\right)$ can be defined without the condidtion that $\mathfrak{X}_{\eta}$ admits a $\mathfrak{X}$-bounded gauge form (cf. [28, Proposition-Definition 7.38]).

Note that the image of $S(\mathfrak{X}, \omega ; T)$ under the forgetful morphism depends only on $\mathfrak{X}_{\eta}$, not on $\mathfrak{X}$ (by Proposition 3.6), and we have

$$
\int_{\mathfrak{X}_{0}} S(\mathfrak{X}, \omega ; T)=\sum_{m \geq 1} \Phi\left(\left[\mathfrak{X}_{\eta}(m), \omega(m)\right]\right) T^{m} \in \mathcal{M}_{k}^{\hat{\mu}}[[T]] .
$$

We then put $S\left(\mathfrak{X}_{\eta}, \widehat{K^{s}}\right):=\int_{\mathfrak{X}_{0}}\left(S\left(\mathfrak{X}, \widehat{K^{s}}\right)\right)=-\lim _{T \rightarrow \infty} \int_{\mathfrak{X}_{0}} S(\mathfrak{X}, \omega ; T) \in \mathcal{M}_{k}^{\hat{\mu}}$ and call it the motivic volume of $\mathfrak{X}_{\eta}$.

Let $K\left(\mathrm{BSRig}_{K}\right)$ be the Grothendieck ring of the category BSRig ${ }_{K}$ of bounded smooth rigid $K$-varieties. The following is an analogue of [19, Proposition 5.5] in the action context. 
Proposition 4.2. There exists a unique homomorphism of groups $\mathrm{MV}: K\left(\operatorname{BSRig}_{K}\right) \rightarrow \mathcal{M}_{k}^{\hat{\mu}}$ such that

$$
\operatorname{MV}([X])=-\lim _{T \rightarrow \infty} \sum_{m \geq 1} \Phi([X(m), \omega(m)]) T^{m},
$$

where $\omega$ is some gauge form on $X$. In particular, if $\mathfrak{X}$ is a generically smooth special formal $R$-scheme, we have $\operatorname{MV}\left(\left[\mathfrak{X}_{\eta}\right]\right)=S\left(\mathfrak{X}_{\eta}, \widehat{K^{s}}\right)$.

4.2. Motivic nearby cycle of a formal function. In this section we consider the special case $R=k[[t]]$ with $k$ a field of characteristic zero (hence we can choose $\varpi=t$ ). Consider a special formal $R$-scheme $\mathfrak{X}$ with a non-constant formal function $\mathfrak{f}: \mathfrak{X} \rightarrow \operatorname{Spf}(R)$ as the structural morphism. A resolution of singularities of $\mathfrak{X}$ exists due to Theorem 2.1. Using the resolution of singularities $\mathfrak{h}: \mathfrak{Y} \rightarrow \mathfrak{X}$ mentioned in the previous section we have the following definition (which was originally introduced by Kontsevich-Soibelman [17] and Lê [19]).

Definition 4.3. The motivic nearby cycle $\mathcal{S}_{\mathfrak{f}}$ of the formal function $\mathfrak{f}: \mathfrak{X} \rightarrow \operatorname{Spf}(R)$ is the following

$$
\mathcal{S}_{\mathfrak{f}}:=\sum_{\emptyset \neq I \subset J}(1-\mathbb{L})^{|I|-1}\left[\widetilde{E}_{I}^{\circ}\right]
$$

It is an element of the Grothendieck ring $\mathcal{M}_{\mathfrak{X}_{0}}^{\hat{\mu}}$. Let us explain why the previous definition of $\mathcal{S}_{\mathfrak{f}}$ is independent of the choice of resolution of singularities of $\mathfrak{X}$ (for more details, see [19, Lemma 5.7]). Observe that after taking the reduction of a formal $k$-scheme, the above construction of the Galois covering with Galois group $\mu_{m_{I}}$ is exactly the construction of DenefLoeser in [11]. In other words, Denef-Loeser [11] use resolution of singularities to formulate an expression of the motivic nearby cycle of a regular function, while base on the resolution of singularities of a special formal scheme $(\mathfrak{X}, \mathfrak{f})$ we use that formula to define the motivic cycles of $\mathfrak{f}$. Therefore, also as in Denef-Loeser [11], the definition of $\mathcal{S}_{\mathfrak{f}}$ does not depend on a particular resolution of singularities of $\mathfrak{X}$.

Definition 4.4. Let $\mathrm{x}$ be a closed point of $\mathfrak{X}_{0}$. Then the motivic Milnor fiber $\mathcal{S}_{\mathfrak{f}, \mathrm{x}}$ of a formal function $\mathfrak{f}$ at $\mathbf{x}$ is the motivic quantity $\left(\{\mathbf{x}\} \hookrightarrow \mathfrak{X}_{0}\right)^{*} \mathcal{S}_{\mathfrak{f}}$ in $\mathcal{M}_{k}^{\hat{\mu}}$.

This definition of $\mathcal{S}_{\mathfrak{f}, \mathbf{x}}$ is compatible with the definition of $\mathcal{S}_{\mathfrak{f}}$ in the following sense. Let $\mathfrak{X} / \mathbf{x}$ be the formal completion of $\mathfrak{X}$ at $\mathbf{x}$. Let $\mathfrak{f}_{\mathbf{x}}: \mathfrak{X} / \mathbf{x} \rightarrow \operatorname{Spf}(R)$ be the formal function induced from $\mathfrak{f}: \mathfrak{X} \rightarrow \operatorname{Spf}(R)$. Then Definition 4.3 can be applied to $\mathfrak{f}_{\mathbf{x}}$, and we have the following

$$
\left(\{\mathbf{x}\} \hookrightarrow \mathfrak{X}_{0}\right)^{*} \mathcal{S}_{\mathfrak{f}}=\mathcal{S}_{\mathfrak{f}_{\mathbf{x}}}=\sum_{\emptyset \neq I \subset J}(1-\mathbb{L})^{|I|-1}\left[\widetilde{E}_{I}^{\circ} \cap \mathfrak{h}_{0}^{-1}(\mathbf{x})\right] .
$$

Summarizing Section 4.1 and Definition 4.3 we get

Corollary 4.5. If $\mathfrak{X}$ is a generically smooth special formal $k[[t]]$-scheme of relative dimension $d$, then the identity

$$
\operatorname{MV}\left(\left[\mathfrak{X}_{\eta}\right]\right)=\mathbb{L}^{-d} \int_{\mathfrak{X}_{0}} \mathcal{S}_{\mathfrak{f}}
$$

holds in $\mathcal{M}_{\kappa}^{\hat{\mu}}$. 
4.3. The integral identity conjecture and geometric part of proof. At this time we have enough materials to state the integral identity conjecture in an exact way. Let $k$ be a field of characteristic zero, and let $(x, y, z)$ be a system of coordinates of the $k$-vector space $k^{d}=k^{d_{1}} \times k^{d_{2}} \times k^{d_{3}}$ in the canonical basis.

Conjecture 4.6 (Kontsevich-Soibelman [17], Conjecture 4.4). Let $f$ be in $k[[x, y, z]]$ invariant by the natural $\mathbb{G}_{m, k}$-action of weight $(1,-1,0)$, with $f(0,0,0)=0$. Let $\mathfrak{X}$ be the formal completion of $\mathbb{A}_{k}^{d}$ along $\mathbb{A}_{k}^{d_{1}}$ with structural morphism $f_{\mathfrak{X}}$ induced by $f$, let $\mathfrak{Z}$ be the formal completion of $\mathbb{A}_{k}^{d_{3}}$ at the origin with structural morphism $f_{\mathfrak{Z}}$ induced by $f(0,0, z)$. Then the following identity

$$
\int_{\mathbb{A}_{k}^{d_{1}}} \mathcal{S}_{f_{\mathfrak{X}}}=\mathbb{L}^{d_{1}} \mathcal{S}_{f_{\mathfrak{Z}}}
$$

holds in $\mathcal{M}_{k}^{\hat{\mu}}$.

Let $\mathfrak{X}$ be as in Conjecture 4.6. Then $\mathfrak{X}$ is a generically smooth special formal $k[[t]]$-scheme of relative dimension $d-1$, and $\mathfrak{X}_{0}=\mathbb{A}_{k}^{d_{1}}$. The generic fiber $\mathfrak{X}_{\eta}$ may be written in the form

$$
\mathfrak{X}_{\eta}=\left\{(x, y, z) \in \mathbb{A}_{\widehat{K^{s}}, \operatorname{Rig}}^{d} \mid \begin{array}{l}
\operatorname{val}(x) \geq 0, \operatorname{val}(y)>0 \\
\operatorname{val}(z)>0, f(x, y, z)=t
\end{array}\right\},
$$

with $K=k((t)), \operatorname{val}(x)=\min _{i}\left\{\operatorname{val}\left(x_{i}\right)\right\}$ and val the standard valuation on the field $\widehat{K^{s}}$. We write $\mathfrak{X}_{\eta}=X_{0} \sqcup X_{1}$, where

$$
\begin{aligned}
& X_{0}=\left\{(x, y, z) \in \mathfrak{X}_{\eta} \mid x=0 \text { or } y=0\right\}, \\
& X_{1}=\left\{(x, y, z) \in \mathfrak{X}_{\eta} \mid x \neq 0 \text { and } y \neq 0\right\} .
\end{aligned}
$$

Theorem 4.7. As identities in $\mathcal{M}_{k}^{\hat{\mu}}, \int_{\mathbb{A}_{k}}{ }_{1} \mathcal{S}_{f_{\mathfrak{X}}}=\mathbb{L}^{d_{1}} \mathcal{S}_{f_{\mathfrak{Z}}}$ and $\operatorname{MV}\left(\left[X_{1}\right]\right)=0$ are equivalent.

Proof. The homogeneity of $f$ implies that provided $x=0$ or $y=0$ we have $f(x, y, z)=$ $f(0,0, z)$, hence we may express $X_{0}$ as a cartesian product $Y_{0} \times Z_{0}$, where

$$
\begin{aligned}
& Y_{0}=\left\{(x, y) \in \mathbb{A}_{\widehat{K^{s}}, \operatorname{Rig}}^{d_{1}+d_{2}} \mid \operatorname{val}(x) \geq 0, \operatorname{val}(y)>0, x=0 \text { or } y=0\right\}, \\
& Z_{0}=\left\{z \in \mathbb{A}_{\widehat{K^{s}}, \operatorname{Rig}}^{d_{3}} \mid \operatorname{val}(z)>0, f(0,0, z)=t\right\} .
\end{aligned}
$$

On the other hand, we may write $Y_{0}=Y_{0,1} \sqcup Y_{0,2}$, with $Y_{0,1}=\left\{x \in \mathbb{A}_{\widetilde{K^{s}}, \operatorname{Rig}}^{d_{1}} \mid 0 \leq \operatorname{val}(x)<\infty\right\}$ and $Y_{0,2}=\left\{y \in \mathbb{A}_{\widetilde{K^{s}}, \operatorname{Rig}}^{d_{2}} \mid \operatorname{val}(y)>0\right\}$. It implies that $X_{0}=\left(Y_{0,1} \times Z_{0}\right) \sqcup\left(Y_{0,2} \times Z_{0}\right)$. Now, by Proposition 4.2,

$$
\operatorname{MV}\left(\left[Y_{0,1} \times Z_{0}\right]\right)=-\lim _{T \rightarrow \infty} \sum_{m \geq 1} \Phi\left(\left[Y_{0,1}(m) \times Z_{0}(m), d x \times \omega(m)\right]\right) T^{m},
$$

where $\omega$ is a certain gauge form on $Z_{0}$ and $d x=d x_{1} \wedge \cdots \wedge d x_{d_{1}}$. By Proposition 3.7,

$$
\Phi\left(\left[Y_{0,1}(m) \times Z_{0}(m), d x \times \omega(m)\right]\right)=\Phi\left(\left[Y_{0,1}(m), d x(m)\right] \cdot \Phi\left(\left[Z_{0}(m), \omega(m)\right]\right) .\right.
$$

Note that $\Phi\left(\left[Y_{0,1}(m), d x(m)\right]=0\right.$, because $Y_{0,1}(m)=\left\{x \in \mathbb{A}_{K(m), \operatorname{Rig}}^{d_{1}} \mid \operatorname{val}(x) \geq 0\right\} \backslash\{0\}$ and $\Phi\left(\left[\left\{x \in \mathbb{A}_{K(m), \operatorname{Rig}}^{d_{1}} \mid \operatorname{val}(x) \geq 0\right\}, d x(m)\right]\right)=\Phi([\{0\}, 1])=1$, hence we get $\operatorname{MV}\left(\left[Y_{0,1} \times Z_{0}\right]\right)=0$.

Also by Proposition 4.2,

$$
\operatorname{MV}\left(\left[Y_{0,2} \times Z_{0}\right]\right)=-\lim _{T \rightarrow \infty} \sum_{m \geq 1} \Phi\left(\left[Y_{0,2}(m) \times Z_{0}(m), d^{\prime} x \times \omega(m)\right]\right) T^{m},
$$


where $\omega$ is a certain gauge form on $Z_{0}$ and $d^{\prime} x=d x_{1} \wedge \cdots \wedge d x_{d_{2}}$. Since $\Phi$ is a morphism of rings (cf. Proposition 3.7),

$$
\Phi\left(\left[Y_{0,2}(m) \times Z_{0}(m), d^{\prime} x \times \omega(m)\right]\right)=\Phi\left(\left[Y_{0,2}(m), d^{\prime} x(m)\right] \cdot \Phi\left(\left[Z_{0}(m), \omega(m)\right]\right) .\right.
$$

A simple computation gives $\Phi\left(\left[Y_{0,2}(m), d^{\prime} x(m)\right]\right)=\mathbb{L}^{-d_{2}}$ for any $m \geq 1$, thus

$$
\operatorname{MV}\left(\left[Y_{0,2} \times Z_{0}\right]\right)=\mathbb{L}^{-d_{2}} \operatorname{MV}\left(\left[Z_{0}\right]\right) ;
$$

and, moreover, this identity also holds in $\mathcal{M}_{k}^{\hat{\mu}}$, by Corollary 4.5 .

As mentioned in Conjecture 4.6, $\mathfrak{Z}$ is the formal completion of $\mathbb{A}_{k}^{d_{3}}$ at the origin with structural morphism $f_{\mathfrak{Z}}$ induced by $f(0,0, z)$; hence it has the relative dimension $d_{3}-1$. Observe that $Z_{0}$ is exactly the generic fiber $\mathfrak{Z}_{\eta}$, thus by Corollary $4.5, \operatorname{MV}\left(\left[Z_{0}\right]\right)=\mathbb{L}^{-d_{3}+1} \mathcal{S}_{f_{\mathfrak{Z}}}$, which induces that $\operatorname{MV}\left(\left[Y_{0,2} \times Z_{0}\right]\right)=\mathbb{L}^{-d_{2}-d_{3}+1} \mathcal{S}_{f_{\mathfrak{Z}}}$ in $\mathcal{M}_{k}^{\hat{\mu}}$, hence

$$
\mathbb{L}^{d_{1}} \mathcal{S}_{f_{\mathfrak{Z}}}=\mathbb{L}^{d-1} \mathrm{MV}\left(\left[X_{0}\right]\right)
$$

in $\mathcal{M}_{k}^{\hat{\mu}}$. Finally, by Corollary $4.5, \int_{\mathbb{A}_{k}^{d_{1}}} \mathcal{S}_{f_{\mathfrak{X}}}=\mathbb{L}^{d-1} \mathrm{MV}\left(\left[\mathfrak{X}_{\eta}\right]\right)$, which completes the proof.

\section{Motivic integration of Hrushovski-Kazhdan}

As shown in Theorem 4.7, the conjectural integral identity is equivalent to $\mathrm{MV}\left(\left[X_{1}\right]\right)=0$ in $\mathcal{M}_{k}^{\hat{\mu}}$. However, in our approach, the foundation of motivic integration for formal schemes is not sufficient to prove the identity $\operatorname{MV}\left(\left[X_{1}\right]\right)=0$. Fortunately, we may find out a solution in motivic integration of Hrushovski-Kazhdan (cf. [13] and [14]) and concrete computations of Hrushovski-Loeser of motivic Milnor fiber (cf. [15]). To apply this theory it is important to suppose that $k$ is an algebraically closed field of characteristic zero, and results in general only hold in $\mathcal{M}_{k, \text { loc }}^{\hat{\mu}}$.

5.1. The theory $\operatorname{ACVF}(0,0)$ and measured categories. Let $\operatorname{ACVF}_{k((t))}(0,0)$ denote the theory of algebraically closed valued fields of equal characteristic zero that extend $k((t))$ (cf. [13]). The theory has two sorts $\mathrm{VF}$ and RV, and one imaginary sort $\Gamma$. The sort VF admits the language of rings. The language of RV consists of abelian group operations ·, /, a unary predicate $\mathbf{k}^{\times}$for a subgroup and a binary operation + on $\mathbf{k}=\mathbf{k}^{\times} \cup\{0\}$. The imaginary sort $\Gamma$ is equipped with a uniquely divisible abelian group. For an algebraically closde valued field of equal characteristic zero $L$, we denote by $R_{L}$ (resp. $\mathfrak{m}_{L}$ ) its valuation ring (resp. the maximal ideal of $\left.R_{L}\right)$. The basis $L$-definable sets of $\operatorname{ACVF}_{k((t))}(0,0)$ are $\operatorname{VF}(L):=L$, $\operatorname{RV}(L):=L^{\times} /\left(1+\mathfrak{m}_{L}\right), \Gamma(L):=L^{\times} / R_{L}^{\times}$and $\mathbf{k}(L):=R_{L} / \mathfrak{m}_{L}$. A definable subset of $\operatorname{VF}^{n}(L)$ is a finite Boolean combination of set of the forms $\operatorname{val}\left(f_{1}\right) \leq \operatorname{val}\left(f_{2}\right)$ or $f_{3}=0$, where $f_{i}$ are polynomials with coefficients in $k((t))$. We also have definable subsets of $\operatorname{RV}^{n}(L), \Gamma^{n}(L)$ and $\mathbf{k}^{n}(L)$ in the same way. There are natural maps between these sets $\mathrm{rv}: \mathrm{VF} \rightarrow \mathrm{RV}$, val $: \mathrm{VF} \rightarrow \Gamma$, val $_{\mathrm{rv}}: \mathrm{RV} \rightarrow \Gamma$ and res $: R_{L} \rightarrow \mathbf{k}(L)$, and also an exact sequence of groups $1 \rightarrow \mathbf{k}^{\times} \rightarrow \mathrm{RV} \stackrel{\text { val }_{\mathrm{rv}}}{\rightarrow} \Gamma \rightarrow 0$.

Let $\mu_{\Gamma} \mathrm{VF}$ be the category of $k((t))$-definable sets (or definable sets, for short) endowed with definable volume forms, up to $\Gamma$-equivalence. One may show that it is graded via the following subcategories $\mu_{\Gamma} \mathrm{VF}[n], n \in \mathbb{N}$. An object of $\mu_{\Gamma} \mathrm{VF}[n]$ is a triple $(X, f, \varepsilon)$ with $X$ a definable subset of $\mathrm{VF}^{\ell} \times \mathrm{RV}^{\ell^{\prime}}$, for some $\ell, \ell^{\prime}$ in $\mathbb{N}, f: X \rightarrow \mathrm{VF}^{n}$ a definable map with finite fibers and $\varepsilon: X \rightarrow \Gamma$ a definable function; a morphism from $(X, f, \varepsilon)$ to $\left(X^{\prime}, f^{\prime}, \varepsilon^{\prime}\right)$ is a definable essential bijection $F: X \rightarrow X^{\prime}$ such that

$$
\varepsilon=\varepsilon^{\prime} \circ F+\operatorname{val}(\operatorname{Jac} F)
$$


away from a proper closed subvariety of $X$. Here, that $F: X \rightarrow X^{\prime}$ is an essential bijection means that there exists a proper closed subvariety $Y$ of $X$ such that $\left.F\right|_{X \backslash Y}: X \backslash Y \rightarrow X^{\prime} \backslash F(Y)$ is a bijection (see [13, Section 3.8]).

Let $\mu_{\Gamma} \mathrm{VF}^{\text {bdd }}$ be the full subcategory of $\mu_{\Gamma} \mathrm{VF}$ whose objects are bounded definable sets with bounded definable forms $\varepsilon$. If considering $\varepsilon: X \rightarrow \Gamma$ as the zero function, we obtain the categories volVF and $\operatorname{volVF}[n]$ as well as volVF ${ }^{\text {bdd }}$ and volVF ${ }^{\text {bdd }}[n]$. In this case, the measure preserving property of a morphism $F$ is characterized by the condition val( $\operatorname{Jac} F)=0$, outside a proper closed subvariety. For simplicity we may omit the symbol $f$ in the triple $(X, f, \varepsilon)$ when no confusion appears.

We also consider the category $\mu_{\Gamma} \mathrm{RV}$ graded by $\mu_{\Gamma} \mathrm{RV}[n]$ for $n \in \mathbb{N}$, defined as follows. An object of $\mu_{\Gamma} \mathrm{RV}[n]$ is a triple $(X, f, \varepsilon)$ with $X$ a definable subset of $\mathrm{RV}^{\ell}$, for some $\ell \in \mathbb{N}$, $f: X \rightarrow \mathrm{RV}^{n}$ a definable map with finite fibers, and $\varepsilon: X \rightarrow \Gamma$ a definable function. A morphism $(X, f, \varepsilon) \rightarrow\left(X^{\prime}, f^{\prime}, \varepsilon^{\prime}\right)$ in $\mu_{\Gamma} \operatorname{RV}[n]$ is a definable bijection $F: X \rightarrow X^{\prime}$ such that

$$
\varepsilon+\sum_{i=1}^{n} \operatorname{val}_{\mathrm{rv}}\left(f_{i}\right)=\varepsilon^{\prime} \circ F+\sum_{i=1}^{n} \operatorname{val}_{\mathrm{rv}}\left(f_{i}^{\prime} \circ F\right)
$$

away from a proper closed subvariety (which is called the measure preserving property). Denote by $\mu_{\Gamma} \operatorname{RES}[n]$ the full subcategory of $\mu_{\Gamma} \mathrm{RV}[n]$ such that, for each object $(X, f, \varepsilon)$, $\operatorname{val}_{\mathrm{rv}}(X)$ is a finite set. The category $\mu_{\Gamma} \mathrm{RV}^{\text {bdd }}$ is defined as $\mu_{\Gamma} \mathrm{RV}$ with val $\mathrm{rv}_{\mathrm{rv}}$-image of objects bounded below. For each object $(X, f, \varepsilon)$ of one of the previous categories, taking $\varepsilon$ being the zero function we shall name the corresponding subcategories by volRV, volRV ${ }^{\text {bdd }}$ and volRES. Let RES be a category defined exactly as volRES except the measure preserving property for morphisms.

Denote by $\mu \Gamma[n]$ the category of pairs $(\Delta, l)$ with $\Delta$ a definable subset of $\Gamma^{n}$ and $l: \Delta \rightarrow \Gamma$ a definable map. A morphism $(\Delta, l) \rightarrow\left(\Delta^{\prime}, l^{\prime}\right)$ of the category is a definable bijection $\lambda: \Delta \rightarrow \Delta^{\prime}$ which is liftable to a definable bijection $\mathrm{val}_{\mathrm{rv}}^{-1} \Delta \rightarrow \mathrm{val}_{\mathrm{rv}}^{-1} \Delta^{\prime}$ such that

$$
|x|+l(x)=|\lambda(x)|+l^{\prime}(\lambda(x)) .
$$

Let $\mu \Gamma^{\text {bdd }}[n]$ be the full subcategory of $\mu \Gamma[n]$ such that, for each object $(\Delta, l)$ of $\mu \Gamma^{\text {bdd }}[n]$, there exists a $\gamma \in \Gamma$ with $\Delta \subset[\gamma, \infty)^{n}$. By definition, the categories $\mu \Gamma$ and $\mu \Gamma^{\text {bdd }}$ are the direct sums $\bigoplus_{n \geq 1} \mu \Gamma[n]$ and $\bigoplus_{n \geq 1} \mu \Gamma^{\mathrm{bdd}}[n]$, respectively. The subcategories whose objects are of the form $(\Delta, 0)$ will be written as $\operatorname{vol} \Gamma$ and $\operatorname{vol} \Gamma^{\text {bdd }}$.

5.2. Hrushovski-Loeser's morphisms generalized. Let $\mathcal{C}$ be one of the categories in the previous subsection. Then, as in [13], we denote the Grothendieck semiring of $\mathcal{C}$ by $K_{+}(\mathcal{C})$ and the associated ring by $K(\mathcal{C})$. By [13], there is a natural morphism of

$$
N: K_{+}\left(\mu \Gamma^{\mathrm{bdd}}\right) \otimes K_{+}\left(\mu_{\Gamma} \mathrm{RES}\right) \rightarrow K_{+}\left(\mu_{\Gamma} \mathrm{VF}^{\mathrm{bdd}}\right)
$$

constructed as follows. Note that two objects admitting a morphism $\lambda$ in $\mu \Gamma^{\text {bdd }}[n]$ define the same element in $K_{+}\left(\mu \Gamma^{\mathrm{bdd}}[n]\right)$, hence $\lambda$ lifts to a morphism in $\mu_{\Gamma} \mathrm{VF}^{\mathrm{bdd}}[n]$ between their pullbacks. Thus there exists a natural morphism $K_{+}\left(\mu \Gamma^{\mathrm{bdd}}[n]\right) \rightarrow K_{+}\left(\mu_{\Gamma} \mathrm{VF}^{\mathrm{bdd}}\right)$ mapping the class of $(\Delta, l)$ to the class of $\left(\mathrm{val}^{-1}(\Delta), l \circ \mathrm{val}\right)$. Also, for each object $(X, f, \varepsilon)$ in $\mu_{\Gamma} \operatorname{RES}[n]$, we may consider an étale map $\ell: X \rightarrow \mathbf{k}^{n}$. By this, we have the natural morphism $K_{+}\left(\mu_{\Gamma} \operatorname{RES}[n]\right) \rightarrow K_{+}\left(\mu_{\Gamma} \mathrm{VF}^{\text {bdd }}\right)$ by sending the class of $(X, f, \varepsilon)$ to the class of $\left(X \times \times_{\ell \text {,res }} R^{n}, \operatorname{pr}_{1} \circ \varepsilon\right)$. In particular, if $X$ is Zariski open in $\mathbf{k}^{n}$, then $X \times_{\ell \text {,res }} R^{n}$ is simply $\operatorname{res}^{-1}(X)$. 
Theorem 5.1 (Hrushovski-Kazhdan [13]). The morphism $N$ is a surjection. Moreover, it also induces a surjective morphism $N$ between the associated rings.

By [13, Proposition 10.10], an element of $K_{+}\left(\mu_{\Gamma} \mathrm{RV}^{\mathrm{bdd}}\right)$ may be written as a finite sum of elements of the form $\left[\left(X \times \mathrm{val}_{\mathrm{rv}}^{-1}(\Delta), f, \varepsilon\right)\right]$. An argument in the proof of [13, Proposition 10.10] also shows that

$$
\left[\left(X \times \operatorname{val}_{\mathrm{rv}}^{-1}(\Delta), f, \varepsilon\right)\right]=\left[\left(X, f_{0}, 1\right)\right] \otimes[(\Delta, l)],
$$

where $f_{0}: X \rightarrow \mathrm{RV}^{n}$ and $l: \Delta \rightarrow \Gamma$ are some definable functions.

Let $! K(\mathrm{RES})$ be the quotient of $K(\mathrm{RES})$ modulo the conditions $\left[\mathrm{val}_{\mathrm{rv}}^{-1}(a)\right]=\left[\mathrm{val}_{\mathrm{rv}}^{-1}(0)\right]$ for $a$ in $\Gamma=\Gamma(\mathbb{Q})$, and $! K(\mathrm{RES})\left[\mathbb{L}^{-1}\right]_{\text {loc }}$ the localization of $! K(\mathrm{RES})\left[\mathbb{L}^{-1}\right]$ with respect to the multiplicative family generated by $1-\mathbb{L}^{i}, i \geq 1$. Let $m, n$ be in $\mathbb{N}, m \geq 1,(\Delta, l)$ in $\mu \Gamma^{\text {bdd }}[n]$ and $e$ in $\Gamma$ with $m e \in \mathbb{Z}$. Set $\Delta(m):=\Delta \cap(1 / m \mathbb{Z})^{n}, \Delta_{l, e}:=l^{-1}(e)$ and

$$
\alpha_{m}(\Delta, l):=\sum_{e \in \mathbb{Z}} \sum_{\gamma \in \Delta_{l, e / m}(m)} \mathbb{L}^{-m|\gamma|-e}(\mathbb{L}-1)^{n} .
$$

By definition $\alpha_{m}(\Delta, l)$ is an element of $! K(\mathrm{RES})\left[\mathbb{L}^{-1}\right]_{\text {loc }}$, and moreover, $\alpha_{m}$ is independent of the choice of coordinates for $\Gamma^{n}$. Indeed, let $\lambda$ be the morphism in $\mu \Gamma^{\text {bdd }}$ from $\left(\Delta_{l, e},\left.l\right|_{\Delta_{l, e}}\right)$ to $\left(\Delta^{\prime}, l^{\prime}\right)$. Then $|\lambda(\gamma)|+l^{\prime}(\lambda(\gamma))=|\gamma|+l(\gamma)=|\gamma|+e$ and the claim follows. Thus $\alpha_{m}$ induces a natural morphism of rings

$$
\alpha_{m}: K\left(\mu \Gamma^{\mathrm{bdd}}\right) \rightarrow ! K(\mathrm{RES})\left[\mathbb{L}^{-1}\right]_{\mathrm{loc}}
$$

By using [15], for any $\widetilde{\Delta}$ in vol $\Gamma^{\text {bdd }}$, one sets $\widetilde{\alpha}_{m}(\widetilde{\Delta}):=\sum_{\gamma \in \widetilde{\Delta}(m)} \mathbb{L}^{-m|\gamma|}(\mathbb{L}-1)^{n}$ and obtains a morphism of rings

$$
\widetilde{\alpha}_{m}: K\left(\operatorname{vol} \Gamma^{\text {bdd }}\right) \rightarrow ! K(\mathrm{RES})\left[\mathbb{L}^{-1}\right]_{\text {loc }} .
$$

It is clear that $\alpha_{m}$ is an extension of $\widetilde{\alpha}_{m}$ and $\alpha_{m}(\Delta, l)=\sum_{e \in \mathbb{Z}} \widetilde{\alpha}_{m}\left(\Delta_{l, e / m}\right) \mathbb{L}^{-e}$.

In order to obtain a morphism $\beta_{m}: K\left(\mu_{\Gamma} \mathrm{RES}\right) \rightarrow ! K(\mathrm{RES})\left[\mathbb{L}^{-1}\right]_{\text {loc }}$ it suffices to define $\beta_{m}([(X, f, 1)])$ for an object $(X, f, 1)$ in $\mu_{\Gamma}$ RES. Assume that $f(X) \subset V_{\gamma_{1}} \times \cdots \times V_{\gamma_{n}}$, i.e., $\operatorname{val}_{\mathrm{rv}}\left(f_{i}(x)\right)=\gamma_{i}$ for every $x$ in $X$. We set $\beta_{m}(X, f, 1):=[X]\left(\mathbb{L}^{-1}[1]_{1}\right)^{m|\gamma|}$ if $m \gamma \in \mathbb{Z}^{n}$ and $\beta_{m}(X, f, 1):=0$ otherwise.

By Hrushovski-Loeser [15], $\operatorname{ker}\left(\widetilde{\alpha}_{m} \otimes \beta_{m}\right)$ contains $\operatorname{ker}\left(N_{0}\right)$, with $N_{0}$ being $N$ reduced to the volume version (for the structure of $\left.K\left(\operatorname{volVF}^{\text {bdd }}\right)\right)$. Similarly, we also have that $\operatorname{ker}\left(\alpha_{m} \otimes \beta_{m}\right)$ contains $\operatorname{ker}(N)$. Thus we obtain morphisms of rings

$$
\widetilde{h}_{m}: K\left(\text { volVF}{ }^{\text {bdd }}\right) \rightarrow ! K(\mathrm{RES})\left[\mathbb{L}^{-1}\right]_{\text {loc }}
$$

and

$$
h_{m}: K\left(\mu_{\Gamma} \mathrm{VF}^{\mathrm{bdd}}\right) \rightarrow ! K(\mathrm{RES})\left[\mathbb{L}^{-1}\right]_{\mathrm{loc}} .
$$

It is shown in [20], Lemma 4.3] that the identity $h_{m}([(X, \varepsilon)])=\sum_{e \in \mathbb{Z}} \widetilde{h}_{m}\left(\left[\varepsilon^{-1}(e / m)\right]\right) \mathbb{L}^{-e}$ holds in $! K(\mathrm{RES})\left[\mathbb{L}^{-1}\right]_{\text {loc }}$.

We also use the morphisms in [15, Section 8.5] with their restriction, namely,

$$
\alpha: K\left(\operatorname{vol} \Gamma^{\mathrm{bdd}}\right) \rightarrow ! K(\mathrm{RES})\left[\mathbb{L}^{-1}\right]
$$

and

$$
\beta: K(\operatorname{volRES}) \rightarrow ! K(\mathrm{RES})\left[\mathbb{L}^{-1}\right]
$$

By definition, $\beta([X])=[X], \alpha([\Delta])=\chi(\Delta)(\mathbb{L}-1)^{n}$ if $\Delta$ is a definable subset of $\Gamma^{n}$, where $\chi$ is the o-minimal Euler characteristic in the sense of [13, Lemma 9.5]. Since $\operatorname{ker}(\alpha \otimes \beta)$ contains in $\operatorname{ker}\left(N_{0}\right)$ (cf. [15]) it gives rise to a morphism of rings

$$
K\left(\operatorname{volVF}^{\mathrm{bdd}}\right) \rightarrow ! K(\mathrm{RES})\left[\mathbb{L}^{-1}\right] .
$$


The composition of it with the localization morphism $! K(\mathrm{RES})\left[\mathbb{L}^{-1}\right] \rightarrow ! K(\mathrm{RES})\left[\mathbb{L}^{-1}\right]_{\mathrm{loc}}$ is denoted by $h$. The following is stated in [20, Proposition 4.4].

Proposition 5.2. The series $Z^{\prime}(X, \varepsilon)(T):=\sum_{m \geq 1} h_{m}([(X, \varepsilon)]) T^{m}$ is a rational function. Moreover, we have $\lim _{T \rightarrow \infty} Z^{\prime}(X, \varepsilon)(T)=-h([X])$.

As in [15, Section 4.3], we define a series $\left\{t_{m}\right\}_{m \geq 1}$ by setting $t_{1}=t, t_{n m}^{m}=t_{n}, n \geq 1$. For a $\mathbf{k}((t))$-definable set $X$ over RES, we may assume $\bar{X} \subset V_{i_{1} / m} \times \cdots \times V_{i_{n} / m}$ for some $n, m$ and $i_{j}$. It is endowed with a natural action $\delta$ of $\mu_{m}$. Now the $\mathbf{k}\left(\left(t^{1 / m}\right)\right)$-definable mapping

$$
\left(x_{1}, \ldots, x_{n}\right) \mapsto\left(x_{1} / \mathrm{rv}\left(t_{m}^{i_{1}}\right), \ldots, x_{n} / \mathrm{rv}\left(t_{m}^{i_{n}}\right)\right)
$$

sends $X$ to a constructible subset $Y$ of $\mathbb{A}_{\mathbf{k}}^{n}$, where $Y$ is endowed with a $\mu_{m}$-action induced from $\delta$. The correspondence $X \mapsto Y$ then defines a morphism $\Theta^{\prime}$ of rings

$$
! K(\mathrm{RES})\left[\mathbb{L}^{-1}\right] \rightarrow ! K_{0}\left(\operatorname{Var}_{k, \hat{\mu}}\right)\left[\mathbb{L}^{-1}\right]
$$

(see [13, Lemma 10.7] and [15, Proposition 4.3.1]). Note that $! K_{0}\left(\operatorname{Var}_{k, \hat{\mu}}\right)$ is the quotient of $K_{0}^{\hat{\mu}}\left(\operatorname{Var}_{k}\right)$ by identifying all the classes $\left[\mathbb{G}_{m}, \sigma\right]$ with $\sigma$ a $\hat{\mu}$-action on $\mathbb{G}_{m}$ induced by multiplication by roots of 1 . Composing $\Theta^{\prime}$ with the natural morphism $! K_{0}\left(\operatorname{Var}_{k, \hat{\mu}}\right)\left[\mathbb{L}^{-1}\right] \rightarrow \mathcal{M}_{k}^{\hat{\mu}}$ induces a morphism of rings $! K(\mathrm{RES})\left[\mathbb{L}^{-1}\right]_{\text {loc }} \rightarrow \mathcal{M}_{k, \text { loc }}^{\hat{\mu}}$, which will be denoted by $\Theta$. Now define $\mathrm{HL}_{m}:=\Theta \circ h_{m}, \widetilde{\mathrm{HL}}_{m}:=\Theta \circ \widetilde{h}_{m}$ and $\mathrm{HL}:=\Theta \circ h$, which are morphisms of rings. As above, we get the identity

$$
\mathrm{HL}_{m}([(X, \varepsilon)])=\sum_{e \in \mathbb{Z}} \widetilde{\mathrm{HL}}_{m}\left(\left[\varepsilon^{-1}(e / m)\right]\right) \mathbb{L}^{-e}
$$

in $\mathcal{M}_{k, \text { loc }}^{\hat{\mu}}$. Moreover, by [20, Proposition 4.6], the series $Z(X, \varepsilon)(T):=\sum_{m \geq 1} \operatorname{HL}_{m}([(X, \varepsilon)]) T^{m}$ is a rational function, and $\lim _{T \rightarrow \infty} Z(X, \varepsilon)(T)=-\mathrm{HL}([X])$.

It is important to recall a comparison result concerning HL and MV, which was proved in [19, Theorem 6.1] and [20, Theorem 4.8]. Let $X$ be a bounded smooth rigid $k((t))^{\text {alg-variety }}$ endowed with a gauge form $\omega$. Then we may consider $(X, \omega)$ as an object $(X, \alpha)$ of the category $\mu_{\Gamma} \mathrm{VF}^{\text {bdd }}$ with $\alpha=$ val $\circ \omega$. For simplicity, an object $(X, f, \alpha)$ of $\mu_{\Gamma} \mathrm{VF}^{\text {bdd }}$ can be simply written as $(X, \alpha)$ when $f$ is clear.

Theorem 5.3. With the previous notation and hypotheses we have loc $(\Phi([X(m), \omega(m)]))=$ $\mathbb{L}^{-d} \mathrm{HL}_{m}([X$, val $\circ \omega])$ and $\operatorname{loc}(\mathrm{MV}([X]))=\mathbb{L}^{-d} \mathrm{HL}([X])$ in $\mathcal{M}_{k, \text { loc }}^{\hat{\mu}}$, where loc denotes the morphism $\mathcal{M}_{k}^{\hat{\mu}} \rightarrow \mathcal{M}_{k, \text { loc }}^{\hat{\mu}}$.

5.3. Proof of the integral identity conjecture for $k=k^{\text {alg }}$. In the present subsection, by using Hrushovski-Kazhdan's motivic integration we show that $\operatorname{HL}\left(\left[X_{1}\right]\right)=0$ in $\mathcal{M}_{k, \text { loc }}^{\hat{\mu}}$ (Theorem 5.5). Then combination of Theorem 5.5 with Theorem 4.7 and Theorem 5.3 will prove the following theorem:

Theorem 5.4. With the previous notation and hypotheses as in Conjecture 4.6 the identity $\int_{\mathbb{A}_{k}^{d_{1}}} \mathcal{S}_{f_{\mathfrak{X}}}=\mathbb{L}^{d_{1}} \mathcal{S}_{f_{\mathfrak{Z}}}$ holds in $\mathcal{M}_{k, \text { loc }}^{\hat{\mu}}$.

Recall that

$$
X_{1}=\left\{\begin{array}{l|l}
(x, y, z) \in \mathbb{A}_{\widehat{K}^{s}, \operatorname{Rig}}^{d} & \begin{array}{l}
\operatorname{val}(x) \geq 0, \operatorname{val}(y)>0, \operatorname{val}(z)>0 \\
x \neq 0, y \neq 0, f(x, y, z)=t
\end{array}
\end{array}\right\} .
$$


We shall consider an alternative definable set

$$
X_{1}^{*}:=\left\{\begin{array}{l|l}
(x, y, z) \in \mathrm{VF}^{d} & \begin{array}{l}
\operatorname{val}(x) \geq 0, \operatorname{val}(y)>0, \operatorname{val}(z)>0 \\
x \neq 0, y \neq 0, \operatorname{rv}(f(x, y, z))=\operatorname{rv}(t)
\end{array}
\end{array}\right\} .
$$

By $\left[20\right.$, Theorem 4.8] we have $\operatorname{HL}\left(\left[X_{1}\right]\right)=\operatorname{HL}\left(\left[X_{1}^{*}\right]\right)$.

Theorem 5.5. For $f$ in Conjecture 4.6, $\mathrm{HL}\left(\left[X_{1}^{*}\right]\right)=0$ in $\mathcal{M}_{k, \text { loc }}^{\hat{\mu}}$.

Proof. Consider the free action of $G:=\mathbb{G}_{m, k((t))}$ alg on $A:=\left(\mathrm{VF}^{d_{1}}-\{0\}\right) \times\left(\mathrm{VF}^{d_{2}}-\{0\}\right) \times \mathrm{VF}^{d_{3}}$ given by $\tau \cdot(x, y, z)=\left(\tau x, \tau^{-1} y, z\right)$ for $\tau \in G$. The canonical projection $A \rightarrow A / G$ then induces a surjection $\rho: X_{1}^{*} \rightarrow \widetilde{X}_{1}^{*}$, with $\widetilde{X}_{1}^{*}$ the image of $X_{1}^{*}$. Note that an element of $\widetilde{X}_{1}^{*}$ is an orbit of the form $\left\{\left(\tau x, \tau^{-1} y, z\right) \mid-\operatorname{val}(x) \leq \operatorname{val}(\tau)<\operatorname{val}(y)\right\}$, which is an annulus analytically isomorphic to $\mathcal{B}(0, r)-\mathcal{B}\left(0, r^{\prime}\right)$ for $r, r^{\prime} \in \Gamma$ with $r^{\prime}-r=\operatorname{val}(x)+\operatorname{val}(y)$, where $\mathcal{B}(0, r)$ is the non-archimedean closed ball centered at 0 of valuative radius $r$. By [19, Lemma 4.1], $\widetilde{X}_{1}^{*}$ is an object in the category volVF ${ }^{\text {bdd }}\left[d_{3}\right]$. For any $\widetilde{\xi} \in \widetilde{X}_{1}^{*}$ and any $(x, y, z) \in \widetilde{\xi}$, the element $\operatorname{val}(x)+\operatorname{val}(y)$ depends only on $\widetilde{\xi}$, not on the representative. Hence the function $\widetilde{\lambda}: \widetilde{X}_{1}^{*} \rightarrow \Gamma_{>0}$, $\widetilde{\xi} \mapsto \operatorname{val}(x)+\operatorname{val}(y)$ if $(x, y, z)$ is in $\widetilde{\xi}$ is well defined. Putting $\widetilde{X}_{1, \gamma}^{*}:=\tilde{\lambda}^{-1}(\gamma) \subset \widetilde{X}_{1}^{*}$, the composition of $\tilde{\lambda}$ with $\rho: X_{1}^{*} \rightarrow \widetilde{X}_{1}^{*}$ yields a definable function $\lambda: X_{1}^{*} \rightarrow \Gamma_{>0}$ such that $\tilde{X}_{1, \gamma}^{*}$ is the image of $X_{1, \gamma}^{*}:=\lambda^{-1}(\gamma)$ under $\rho$. For any $\gamma \in \Gamma_{>0}$, every fiber of $\left.\rho\right|_{X_{1, \gamma}^{*}}$ is analytically isomorphic to $A_{\gamma}:=\{u \in \mathrm{VF} \mid 0 \leq \operatorname{val}(u)<\gamma\}$ as annuli of the same modulus $\gamma$. Hence we have the identity $\left[X_{1, \gamma}^{*}\right]=\left[\widetilde{X}_{1, \gamma}^{*}\right]\left[A_{\gamma}\right]$ in $K\left(\operatorname{volVF}{ }^{\text {bdd }}\right)$.

Let us now consider the function $\nu: \Gamma_{>0} \rightarrow \mathcal{M}_{k, \text { loc }}^{\hat{\mu}}$ which is given by $\nu(\gamma)=\operatorname{HL}\left(\left[X_{1, \gamma}^{*}\right]\right)$. It is proved that $\nu$ is a definable function in the sense of [19, Lemma 4.2], and in that setting $\operatorname{HL}\left(\left[X_{1}^{*}\right]\right)=\int_{\gamma \in \Gamma_{>0}} \nu(\gamma) d \chi$. Since $\left[X_{1, \gamma}^{*}\right]=\left[\widetilde{X}_{1, \gamma}^{*}\right]\left[A_{\gamma}\right]$ and HL is a morphism of rings, we get $\nu(\gamma)=\operatorname{HL}\left(\left[\widetilde{X}_{1, \gamma}^{*}\right]\right) \cdot \operatorname{HL}\left(\left[A_{\gamma}\right]\right)$. Write $\gamma=p / q$ with $(p, q)=1$. By the construction of $\widetilde{\mathrm{HL}}_{m}$, if $m$ is not divisible by $q$, then $\widetilde{\mathrm{HL}}_{m}\left(\left[A_{p / q}\right]\right)=0$. If $m=l q$ for $l \in \mathbb{N}_{>0}$, we have

$$
\widetilde{\mathrm{HL}}_{l q}\left(\left[A_{p / q}\right]\right)=\left[\left\{\varphi(t) \in k[t] /\left(t^{2 l q+1}\right) \mid 0 \leq \operatorname{ord}_{t} \varphi(t)<m p\right\}\right] \mathbb{L}^{-2 l q+1}=\mathbb{L}-\mathbb{L}^{-l r+1},
$$

with $r=\min \{p, 2 q\}$. It thus implies from Proposition 5.2 that

$$
\mathrm{HL}\left(\left[A_{p / q}\right]\right)=-\lim _{T \rightarrow \infty} \sum_{l \geq 1} \widetilde{\mathrm{HL}}_{l q}\left(\left[A_{p / q}\right]\right) T^{l q}=-\mathbb{L} \lim _{T \rightarrow \infty}\left(\frac{T^{q}}{1-T^{q}}-\frac{\mathbb{L}^{-r} T^{q}}{1-\mathbb{L}^{-r} T^{q}}\right)=0 .
$$

This means that $\nu(\gamma)=0$ for any $\gamma \in \Gamma_{>0}$, hence $\operatorname{HL}\left(\left[X_{1}^{*}\right]\right)=0$ in $\mathcal{M}_{k, \text { loc }}^{\hat{\mu}}$.

Remark 5.6. Recently, by using Cluckers-Loeser's motivic integration [5] we have provided a new proof of the integral identity conjecture (in the framework of $\mathcal{M}_{k, \text { loc }}^{\hat{\mu}}$ ) for any field $k$ of characteristic zero (cf. [21]).

\section{REFERENCES}

[1] K. Behrend, Donaldson-Thomas invariants via microlocal geometry, Ann. of. Math. 170 (2009), no. 3, 1307-1338.

[2] V. Berkovich, Vanishing cycles for formal schemes II, Invent. Math. 125(2) (1996), 367-390.

[3] P. Berthelot, Cohomologie rigide et cohomologie rigide à supports propres, Prépublication, Inst. Math. de Rennes 1996.

[4] F. Bittner, On motivic zeta functions and the motivic nearby fiber, Math. Z. 249 (2005), 63-83.

[5] R. Cluckers, F. Loeser, Constructible motivic functions and motivic integration, Invent. Math. 173 (2008), no. 1, 23-121. 
[6] B. Conrad, Irreducible components of rigid spaces, Ann. Inst. Fourier (Grenoble) 49 (1999), no. 2, $473-541$.

[7] A. J. de Jong, Crystalline Dieudonné module theory via formal and rigid geometry, Inst. Hautes Études Sci. Publ. Math. 82 (1996), 5-96.

[8] J. Denef, F. Loeser, Motivic Igusa zeta functions, J. Algebraic Geom. 7 (1998), 505-537.

[9] J. Denef, F. Loeser, Motivic exponential integrals and a motivic Thom-Sebastiani theorem, Duke Math. J. 99 (1999), no. 2, 285-309.

[10] J. Denef, F. Loeser, "Geometry on arc spaces of algebraic varieties" in European congress of Mathematics, Vol. 1 (Barcelona, 2000), Progr. Math. 201, Birkhaüser, Basel, 2001, 327-348.

[11] J. Denef, F. Loeser, Lefschetz numbers of iterates of the monodromy and truncated arcs, Topology 41 (2002), no. 5, 1031-1040.

[12] M. J. Greenberg, Schemata over local rings, Ann. of Math. 73 (1961), 624-648.

[13] E. Hrushovski, D. Kazhdan, Integration in valued fields, in Algebraic and Number Theory, Progress in Mathematics 253, 261-405 (2006), Birkh äuser.

[14] E. Hrushovski, D. Kazhdan, The value ring of geometric motivic integration, and the Iwahori Heck algebra of $S L_{2}$. With an appendix by Nir Avni, Geom. Funct. Anal. 17 (2008), 1924-1967.

[15] E. Hrushovski and F. Loeser, Monodromy and the Lefschetz fixed point formula, Ann. Sci. École Norm. Sup. 48 No. 2 (2015), 313-349.

[16] D. Joyce, Y. Song, A theory of generalized Donaldson-Thomas invariants, Mem. Amer. Math. Soc. 217 (2012).

[17] M. Kontsevich, Y. Soibelman, Stability structures, motivic Donalson-Thomas invariants and cluster tranformations, arXiv: 0811.2435vl.

[18] M. Kontsevich, Y. Soibelman, Motivic Donaldson-Thomas invariants: summary of results, in Mirror Symmetry and Tropical Geometry, Contemporary Mathematics 527 (Ed. R. Castano-Bernard, Y. Soibelma and I. Zharkov), Amer. Math. Soc., Providence, RI, (2010), 55-90

[19] Q.T. Lê, Proofs of the integral identity conjecture over algebraically closed fields, Duke Math. J. 164 No. 1 (2015), 157-194.

[20] Q. T. Lê, The motivic Thom-Sebastiani theorem for regular and formal functions, to appear in J. reine angew. Math., DOI: 10.1515/crelle-2015-0022, URL: http://www.degruyter.com/view/j/crll.ahead-ofprint/crelle-2015-0022/crelle-2015-0022.xml?format=INT; arXiv:1405.7065.

[21] Q. T. Lê, A proof of the integral identity conjecture, II, preprint, arXiv:1508.00425.

[22] F. Loeser, J. Sebag, Motivic integration on smooth rigid varieties and invariants of degenerations, Duke Math. J. 119 (2003), no. 2, 315-344.

[23] E. Looijenga, Motivic measures, Astérisque 276 (2002), 267-297, Séminaire Bourbaki 1999/2000, no. 874.

[24] J. Nicaise, J. Sebag, Motivic Serre invariants, ramification, and the analytic Milnor fiber, Invent. Math. 168 (2007), no. 1, 133-173.

[25] J. Nicaise, J. Sebag, Motivic Serre invariants of curves, Manuscripta Math. 123 (2007), no. 2, $105-132$.

[26] J. Nicaise, J. Sebag, Motivic Serre invariants and Weil restriction, J. Algebra. 319 (2008), 1585-1610.

[27] J. Nicaise, Formal and rigid geometry: an intuitive introduction and some applications, Enseign. Math. 54(2) (2008), no. 3-4, 213-249.

[28] J. Nicaise, A trace formula for rigid varieties, and motivic Weil generating series for formal schemes, Math. Ann. 343 (2009), 285-349.

[29] J. Sebag, Intégration motivique sur les schémas formels, Bull. Soc. Math. France 132 (2004), no. 1, 1-54, Séminaire Bourbaki 1999/2000, no. 874.

[30] M. Temkin, Desingularization of quasi-excellent schemes in characteristic zero, Adv. Math. 219 (2008), no. $2,488-522$.

[31] R. P. Thomas, A holomorphic Casson invariant for Calabi-Yau 3-folds, and bundles on K3 fibrations, J. Differential Geom. 54 (2000), no. 2, 367-438, Séminaire Bourbaki 1999/2000, no. 874.

Department of Mathematics, Vietnam National University

334 Nguyen Trai Street, Thanh Xuan District, Hanoi, Vietnam

E-mail address: leqthuong@gmail.com

BCAM - Basque Center for Applied Mathematics

Alameda de Mazarredo 14, E-48009 Bilbao, Basque Country, Spain

E-mail address: qle@bcamath.org 